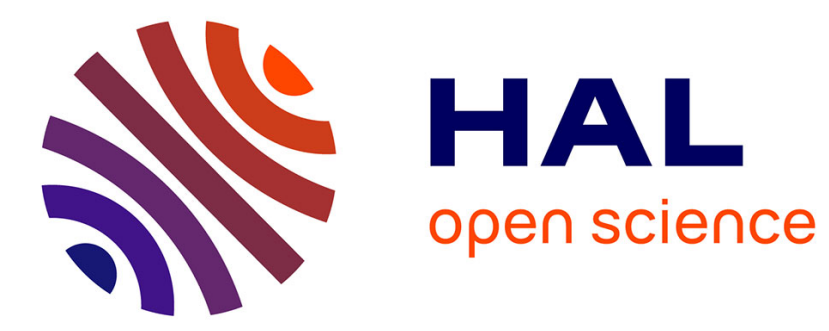

\title{
Computer-aided mobility analysis of parallel mechanisms
}

Xiaorong Zhu, Huiping Shen, Chengqi Wu, Damien Chablat, Tingli Yang

\section{To cite this version:}

Xiaorong Zhu, Huiping Shen, Chengqi Wu, Damien Chablat, Tingli Yang. Computer-aided mobility analysis of parallel mechanisms. Mechanism and Machine Theory, 2020, 148, pp.103810. 10.1016/j.mechmachtheory.2020.103810 . hal-02477808

\section{HAL Id: hal-02477808 \\ https://hal.science/hal-02477808}

Submitted on 13 Feb 2020

HAL is a multi-disciplinary open access archive for the deposit and dissemination of scientific research documents, whether they are published or not. The documents may come from teaching and research institutions in France or abroad, or from public or private research centers.
L'archive ouverte pluridisciplinaire $\mathbf{H A L}$, est destinée au dépôt et à la diffusion de documents scientifiques de niveau recherche, publiés ou non, émanant des établissements d'enseignement et de recherche français ou étrangers, des laboratoires publics ou privés. 


\title{
Computer-aided Mobility Analysis of Parallel Mechanisms
}

\author{
Xiaorong Zhu, E-mail:zxr@cczu.edu.cn \\ School of Mechanical Engineering, Changzhou University, 213164 Jiangsu, China \\ Huiping Shen*,E-mail:shp65@126.com \\ School of Mechanical Engineering, Changzhou University, 213164 Jiangsu, China
}

Chengqi Wu, E-mail:753272704@qq.com

School of Mechanical Engineering, Changzhou University, 213164 Jiangsu, China

Damien Chablat, E-mail:damien.chablat@cnrs.fr

Laboratoire des Sciences du Numérique de Nantes, UMR 6004 Nantes, France

Tingli Yang, E-mail:yangtl@126.com

School of Mechanical Engineering, Changzhou University, 213164 Jiangsu, China

* Corresponding author

\begin{abstract}
To determine mobility properties is one of the most challenging issue in the analysis and synthesis of parallel manipulators (PMs). However, currently, the most used methods mainly rely on experiences and manual analysis, which led to inefficient implementation. The motivation of this paper is to present an automatic mobility analysis algorithm and software package for the researchers and designers with an effective and practical means. According to the topological design theory of PMs based on position and orientation characteristic (POC) equations, this paper proposes a set of computer algorithmic rules and procedures for automated mobility analysis of PMs in the most user-friendly and efficient way. Firstly, a complete digital information model for topological structures which has a mapping relationship with the POC of a PM is proposed. This model not only describes the dimension of the motion outputs, but also includes the mapping relationship between the output orientation and the axes of the kinematic joints. Secondly, algorithmic rules are established that convert the union and intersection operations of POC into binary logical operations and then the detailed algorithmic procedures for an automatic mobility analysis are presented. In what follows, a corresponding software for automatic mobility analysis is described. The software package is equipped with a GUI that facilitates the input and allows the visualization of the results. Finally, four typical examples are provided to show the effectiveness of the software package for most of parallel mechanisms (not including some paradoxical mechanisms).
\end{abstract}

Keywords: mobility analysis; degree of freedom; position and orientation characteristic; parallel mechanisms 


\section{Introduction}

Nowadays, parallel manipulators or mechanisms (in short, PMs) can be found in industrial machines because of important mechanical and kinematic properties over their serial counterparts[1], such as higher stiffness, better load weight ratio, lower inertia, higher accuracy. In their design, mobility analysis is a fundamental issue for the research on mechanical synthesis, kinematic and dynamic analysis. Here, mobility analysis includes the calculation of the number of degree of freedom (in short, DOF) and the evaluation the output motion properties of a manipulator.

It is well known that the conventional mobility analysis based on the Chebyshev-Grubler-Kutzbach (CGK) [2] criterion fails in some PMs, this is due to neglecting the geometrical relationships between joints axes. In recent years, different approaches to the mobility analysis of PMs have been studied systematically, and various formulas and methods have been derived and presented [3-26]. But many of these formulas and methods may be reducible to the same original formula and the existing methods can be grouped into four categories: methods based on screw theory [5-12], methods based on Lie group theory [13-17], methods based on the theory of Position and Orientation Characteristics (POC) [18-23], and methods based on linear transformations [2,24].

Most of existing methods for mobility analysis of PMs are performed manually. This task is often complex and difficult because much experience is required to deal with dependency or deep mathematical knowledge is required to establish the kinematic model. An advanced algorithm for the automatic mobility analysis of PMs is an effective solution to afore mentioned problems. However, as far as the authors of the paper know, computer-aided mobility analysis can be found only in very few papers $[12,13,17,29,30]$. In order to compare the feasibility of computerization of mobility analysis for PMs, the principle and characteristics of the existing relevant methods are listed in Table 1.

The approach using screw theory has been successfully applied in many PMs. The computation of the degree of freedom utilizes the reciprocal relation between twist and wrench defined by the mechanical constraints [5-11], and mainly involves the linear solution of systems of screws. In this sense, this method has some potential for automatic analysis. However, it is difficult to obtain the screw system automatically for the average designer. Cao [12] took the first joint of a leg as a reference to establish the system of all screws of the leg, and realized an automatic analysis of the mobility of symmetrical PMs.

The mobility analysis using Lie Group theory is based on the "multiplication" operation and the "intersection" operation of Euclidean subgroups [13-16]. There are too many operation rules involved in this process (over 107 rules [14] ). This method is therefore not suitable to be implemented directly in an automated algorithm. By translating group theory and subsets and subgroups into the Lie algebra and its vector subspaces and its subalgebras, a computer-aided method for mobility analysis was proposed by Daniel[17]. But the datum describing the infinitesimal screws associated with each kinematic pair of the kinematic chain in an arbitrary position should be entered manually.

The method based on the theory of linear transformations, relates the number of degree of freedom of a chain or a PM to the rank of the Jacobian matrix. Using this method, Angeles and Gosselin [24] obtained the mobility of several over-constraint mechanisms including the Bennet mechanism. But the Jacobian matrix, which maps a vector in joint velocity space into external velocity space, needs to develop the kinematic equations. From this point, this method is quite complicated, and to the best of the authors' knowledge, there exist no general methods that can be automatically used for the PMs.

With the last twenty years, algebraic methods have become successful in solving problems in mechanism analysis and synthesis, and it is also called GA-based method. Husty and Schröcker [25] used methods from algebraic geometry to define the DOF of a PM as Hilbert Dimension of a set of nonlinear polynomial kinematic constraint equations. By introducing Clifford algebra, Huo et al., [26,27] proposed an analytical approach for the determination of the motions/constraints, mobility and singularity of PMs. Based on the screw theory and the Chasles' theorem, Yang [28] presented a method for the type synthesis of 3T1R PMs with variable rotational axis. One main advantage of the GA-based method is that subspace can be added, subtracted, and intersected in its framework, which is beneficial to be carried out in automatic way. However, the establishment of algebraic forms or algebraic equations needs the designers to have the abstruse mathematical knowledge.

So-called POC theory for PMs means the topological design theory for PMs based on Position and Orientation Characteristics (POC) equations [18]. The mobility analysis method based on the POC theory, has clear formulas and 
judgment criteria that are easy to use and program. To solve the mobility analysis problems, based on POC theory, a computer-aided software tool for the mobility analysis of PMs will be implemented in this work. The initial digital model and the revelent algorithms of mobility analysis were investigated in $[29,30]$ for the PMs constituted only by revolute joints and prismatic joints. Considering the geometric properties of multi-DOF pairs and the coupling between rotations and translations, this paper presents a complete representation of topological structure of PMs, and gives the systematic rules for performing the union and intersection operations of POC. Furthermore, the algorithms of mobility analysis for serial and parallel mechanisms are proposed. Finally, a software package for automatic mobility analysis is described and some typical examples of PMs are presented to illustrate its effectiveness.

Table 1 The methods of mobility analysis and their main features

\begin{tabular}{|c|c|c|c|c|}
\hline Methods & $\begin{array}{l}\text { Traditional CGK } \\
\text { criterion }\end{array}$ & Based on screw theory & $\begin{array}{l}\text { Based on displacement } \\
\text { Subgroup/Submanifold }\end{array}$ & Based on POC set \\
\hline Math tool & --- & Screw algebra & Lie algebra & Set theory \\
\hline Representation & Real number & $\begin{array}{l}\text { Motion/constraint screw } \\
\text { system }\end{array}$ & $\begin{array}{l}\text { Symbol of subgroup } \\
\text { /submanifold }\end{array}$ & $\begin{array}{l}\text { POC Set } \\
\text { Number of DOF }\end{array}$ \\
\hline DOF formula & $F=\sum_{i=1}^{m} f_{i}-d(m-n+1)$ & $\begin{array}{l}F=d(n-g-1)+\sum_{i=1}^{g} f_{i}+v \\
v=\sum_{i=1}^{p} g_{i}-\lambda p-k\end{array}$ & $\begin{aligned} F= & \sum_{i=1}^{m} f_{i}-\sum_{j=1}^{(n+1)} \operatorname{dim}\left(A_{j}^{m l f}\right) \\
& +\operatorname{dim}\left(\sum_{j=1}^{(n+1)} A_{j}^{m l f}\right)\end{aligned}$ & $\begin{array}{l}F=\sum_{i=1}^{m} f_{i}-\sum_{j=1}^{(v+1)} \xi_{L j} \\
\xi_{\mathrm{L}_{j}}=\operatorname{dim} \cdot\left(\left(\mathrm{I}_{\mathrm{i}=1}^{\mathrm{j}} \mathrm{M}_{\mathrm{b}_{\mathrm{j}}}\right) \mathrm{UM}_{\mathrm{b}_{(j+1)}}\right)\end{array}$ \\
\hline Algorithm & Elementary algebra & $\begin{array}{l}\text { Operations of screw system: } \\
\text { - Linear operations } \\
\text { - Independence criterion of } \\
\text { screw systems }\end{array}$ & $\begin{array}{l}\text { Operation of displacement } \\
\text { subgroup } \\
\text { - Union operations (107) } \\
\text { - Intersection operations } \\
\text { (107) }\end{array}$ & $\begin{array}{l}\text { Operation of POC set } \\
\text { - Union operations ( } 8 \\
\text { linear, } 2 \text { nonlinear) } \\
\text { - Intersection operations } \\
\text { (12 linear, } 2 \text { nonlinear) }\end{array}$ \\
\hline Main featrues & $\begin{array}{l}\diamond \quad \text { Easy to perform } \\
\diamond \quad \text { Fail to most } \\
\text { spatial PMs }\end{array}$ & $\begin{array}{ll}\diamond \quad \text { Simple linear operations } \\
\diamond \quad \text { Local DOF } \\
\diamond \quad \text { Need verification of full- } \\
\text { cycle DOF } \\
\diamond \quad \text { Including paradoxical } \\
\quad \text { mechanisms }\end{array}$ & $\begin{array}{ll}\diamond \text { Too many rules } \\
\diamond \text { Complicated math tool } \\
\diamond \text { Full-cycle DOF } \\
\diamond \text { Excluding paradoxical } \\
\text { mechanisms }\end{array}$ & $\begin{aligned} \diamond & \text { Simple linear } \\
& \text { operations } \\
\diamond & \text { Fewer rules } \\
\diamond & \text { Full-cycle DOF } \\
\diamond & \text { Excluding paradoxical } \\
& \text { mechanisms }\end{aligned}$ \\
\hline
\end{tabular}

Table 1 (Continued)

\begin{tabular}{|c|c|c|c|}
\hline Methods & Based on linear & \multicolumn{2}{|c|}{ Based on GA } \\
\hline Math tool & Linear algebra & Study's kinematic mapping & Conformal geometric algebra \\
\hline Representation & Rank of Jacobian matrix & $\begin{array}{l}\text { Dimension of local tangent spaces of } \\
\text { kinematic varieties }\end{array}$ & Linear-independent constraints \\
\hline DOF formula & $F=\sum_{i=1}^{m} f_{i}-\sum_{j=1}^{k} \operatorname{dim}\left(R_{A j}\right)+\operatorname{dim}\left(\mathbb{I}_{j=1}^{k} R_{A j}\right)$ & Hilbert dimension & $\begin{array}{l}\text { Number of linear-independent } \\
\text { constraints }\end{array}$ \\
\hline Algorithm & Linear algebra & Study's kinematic mapping & $\begin{array}{l}\text { Inner product, Outer product, } \\
\text { Dual product, Shuffle product }\end{array}$ \\
\hline
\end{tabular}




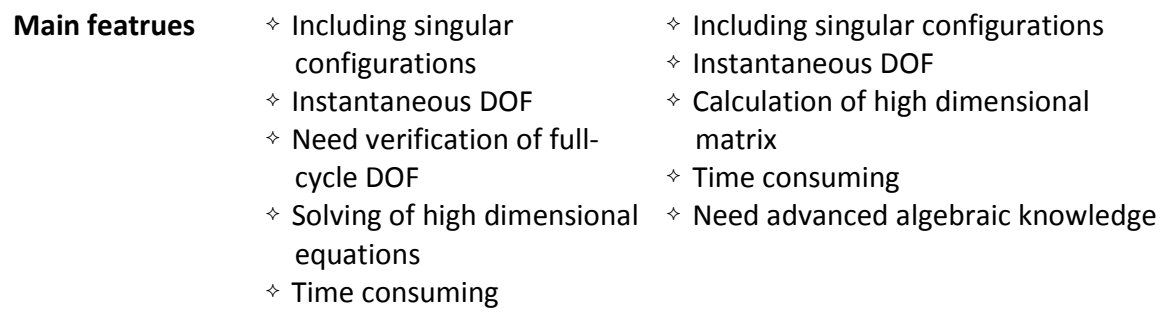

$\diamond$ Including singular configurations

$\diamond$ Instantaneous DOF

$\diamond$ Calculation of high dimensional matrix

$\diamond$ Time consuming

$\diamond$ Need advanced algebraic knowledge

$\diamond$ Simple operations

$\diamond$ Including singular

configurations

$\diamond \quad$ Instantaneous DOF

$\diamond$ Need advanced algebraic knowledge

It should be pointed out that the software package described in this paper currently could not analyze some "paradoxical" mechanisms, for example Bennett and Bricard mechanisms since the mobility of these mechanisms is not only related to their geometric topological structure, but also related to the dimensional constraint parameters (i.e., link length and skew angle comply with a certain functional relationship) which can not be expressed in the matrices of topological structure proposed here.

\section{POC theory for PMs}

This section briefly introduces some basics of POC theory for PMs, which are necessary in the mobility analysis of PMs.

\subsection{Definition and related equations of POC}

\subsubsection{Definition of $P O C$}

To describe the relative motion characteristics between any two components in a mechanism, the POC is introduced in [18] as

$$
\begin{aligned}
& M=\left(\begin{array}{c}
\left.\mathrm{t}^{\xi_{11}}(\text { dir. })+\left\{\mathrm{t}^{\xi_{12}} \text { (dir. }\right)\right\} \\
r^{\xi_{\mathrm{r} 1}}(\text { dir. })+\left\{\mathrm{r}^{\xi_{\mathrm{r}}}(\text { dir. })\right\}
\end{array}\right) \\
& \xi=\xi_{\mathrm{t} 1}+\xi_{\mathrm{r} 1} \leq \mathrm{F}
\end{aligned}
$$

where

$\mathrm{M}$-- POC of the output motion link of the PM.

$\mathrm{t}^{\xi t 1}$ (dir.) -- Independent output translation elements.

$\mathrm{t}^{\{\mathrm{t} 2}$ (dir.) -- Non-independent output translation elements.

$r^{\text {fr1 }}$ (dir.) -- Independent output rotation elements.

$r^{\xi r 2}$ (dir.) -- Non-Independent output rotation elements.

$\xi_{t 1}\left(\xi_{r 1}\right)$-- Number of the independent output translation (rotation) elements $\left(\xi_{t 1}, \xi_{r 1}=0,1,2\right.$ or 3$)$.

(dir.) -- Direction of output translation (or rotation) with respect to joints axes.

$\xi$-- Number of independent output elements in the POC.

F -- DOF of the mechanism.

For most mechanisms, the non-independent output element is constant (excluding the singular configurations) and therefore not considered.

\subsubsection{POC equation for serial mechanism}

The POC of a serial leg is expressed as

$$
\mathrm{M}_{\mathrm{L}}=\mathrm{U}_{\mathrm{i}=1}^{\mathrm{m}} \mathrm{M}_{\mathrm{Ji}}
$$

where

$M_{L}--P O C$ of the output motion link (e.g. end link of the serial leg).

$\mathrm{M}_{\mathrm{ji}}-\mathrm{POC}$ of the $\mathrm{i}^{\text {th }}$ joint of the serial leg.

$\mathrm{m}$-- Number of joints in the serial leg. 
U- "Union" operation [18].

\subsubsection{POC equation for parallel mechanism}

The POC of a parallel mechanism is

$$
M_{p a}=I_{j=1}^{n} M_{L j}
$$

where

$M_{p a}$-- POC of the output motion link (e.g. moving platform of the PM).

$\mathrm{n}$-- Number of serial legs of the PM.

$\mathrm{M}_{\mathrm{Lj}}$-- POC of the $\mathrm{j}^{\text {th }}$ leg referred to the same base point.

$\cap$-- "Intersection" operation [18].

\subsection{Equation of mobility}

A PM with $(v+1)$ serial legs can be considered as a combination of $v$ independent single-loop-chains (in short, SLC). The structure composition of a PM based on SLC [18] is shown in Fig.1. Two serial legs are chosen to form the first independent loop (named as $\mathrm{SLC}_{1}$ ), and the moving platform is considered as an active component of this loop. Then, $\mathrm{SLC}_{1}$ is regarded as a whole (i.e., an equivalent sub-PM), and combined with another leg to form a second independent loop (named as $\left.\mathrm{SLC}_{2}\right)$; and in the same way, the $(v+1)^{\text {th }}$ leg is combined with $\operatorname{SLC}_{(v-1)}$ to form the $v^{\text {th }}$ independent loop (named as SLC ).

According to the theory based on POC, the number of the mobility F, i.e., the DOF of a PM can be calculated by Eq.(5), and the property of mobility can be evaluated by POC of the moving platform with respect to the fixed platform.

$$
F=\sum_{i=1}^{m} J_{i}-\sum_{j=1}^{v} \xi_{L_{j}}=\sum_{j=1}^{v+1} f_{j}-\sum_{j=1}^{v} \xi_{L_{j}}
$$

where,

$$
\begin{aligned}
& \xi_{L_{j}}=\operatorname{dim} .\left(\left(I_{i=1}^{j} M_{L_{i}}\right) U M_{L_{(j+1)}}\right) \\
& v=m-n+1
\end{aligned}
$$

where

F -- DOF of the PM.

$\mathrm{J}_{\mathrm{i}}-$ - DOF of the $\mathrm{i}^{\text {th }}$ joint in the PM.

$\mathrm{m}$-- Number of joints in the PM.

$\xi_{\mathrm{Lj}}--$ Number (or rank) of the independent displacement equations of the $\mathrm{j}^{\text {th }}$ independent loop.

$v$-- Number of the independent loops of the PM.

$f_{j}--$ DOF of all joints of the $j^{\text {th }}$ leg.

$\mathrm{n}$-- Number of components of the PM.

$I_{i=1}^{j} M_{L i}-$ POC of the equivalent sub-PM composed by the first $j$ legs.

$M_{L(j+1)}$-- POC of the $(j+1)^{\text {th }}$ leg.

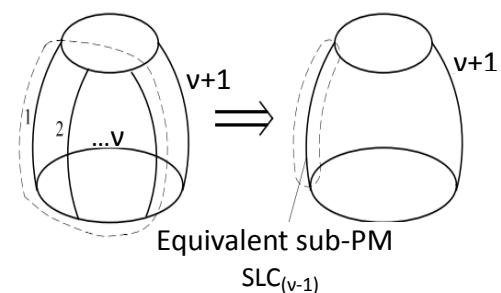


Fig. 1. Basic independent loops composed of PMs

\subsection{Key steps for computer-aided mobility analysis}

(1) The topological description of PM is one of the most significant issues in the analysis of a PM. It should include the complete topological information of the PM with the most concise representation so that it can be recognized and extracted automatically.

(2) POC description for a leg and for a PM is a fundamental problem in automatic mobility analysis. Although the symbolic description as in Eq.(1) is good for expressing the geometric meaning, it is difficult to realize the transitivity of the joint orientation relation.

(3) The most critical issue is how to establish algorithmic rules for POCs suitable for computer programming to implement the "union" and "intersection" operations. In essential, these operations for POCs mainly involve determining spatial linear independency or not.

\section{Representation of a PM and its POC}

Generally, a PM consists of a moving platform connected to a fixed platform by several serial legs, and its mobility (including number and property) is completely affected by the types of kinematic joints and the geometrical relationships between the joint axes. Thus, the topology definition of a PM must be carried out before analyzing the mobility.

First of all, it needs to be emphasized that in the following the legs of the studied PMs do not include redundant kinematic pairs.

The pair types considered in the digital topological description of PMs are only 1-DOF (i.e. $f_{i}=1$ ) revolute $(R)$ and prismatic (P) pair, as any multi-DOF kinematic pair can be produced as a combination of $R$ and $P$ pairs. For instance, universal (U), spherical (S) and cylindrical (C) pairs can be regarded as combinations of two $R$ pairs with orthogonal axes, three $R$ pairs with concurrent axes, and one $R$ pairt and one $P$ pair with collinear axes, respectively.

Mobility analysis of a PM (not includign some paradoxical mechanisms) deals only with the dimensional constrained types, i.e. the geometrical relationships between joints axes. In a serial leg or between legs, there are six basic geometrical relationships proposed by the authors in [18] as: parallel, orthogonal, coaxial, spatial common point, coplanar and arbitrary, here denoted symbolically using "II", “ $\perp$ ", “|", “*”, "\#” and "-", respectively.

\subsection{Digital representation of a serial leg and its POC}

\subsubsection{Digital matrix of serial leg}

As described in [30], a digital ordered topological structure matrix representation is proposed including not only what type of pairing the joint has, but also what pair of orientations the joint characterizes. The ordered topology matrix (L) of a serial leg is expressed as an $f \times f$ matrix, where $f$ is the number of 1-DOF joints in the leg.

$$
\mathrm{L}=\left[\begin{array}{lllll}
\mathrm{J}_{1} & \mathrm{~L} & N_{\mathrm{li}} & \mathrm{L} & N_{1 \mathrm{f}} \\
\mathrm{M} & \mathrm{O} & & & \mathrm{M} \\
N_{\mathrm{i} 1} & & \mathrm{~J}_{\mathrm{i}} & & N_{\mathrm{if}} \\
\mathrm{M} & & & \mathrm{O} & \mathrm{M} \\
N_{\mathrm{f} 1} & \mathrm{~L} & N_{\mathrm{fi}} & \mathrm{L} & \mathrm{J}_{\mathrm{f}}
\end{array}\right]
$$

The diagonal element $J_{i}(i=1 \sim f)$ denotes the type of joint $i$ labeled from the fixed platform to the moving platform in sequence. $\mathrm{J}_{1}$ is connected to the fixed platform and $\mathrm{J}_{\mathrm{f}}$ is connected to the moving platform.

The non-diagonal element $N_{\mathrm{ij}}(\mathrm{i}, \mathrm{j}=1 \sim \mathrm{f})$ represents the geometrical relationship between joint $\mathrm{i}$ and joint $\mathrm{j}$ ( 6 kinds of geometric relationship above-mentioned). So, the topological structure matrix $L$ is symmetrical.

For convenience of programming, each element of matrix $L$ is expressed numerically so that the ordered topological structure matrix can be handled mathematically. Here, $N_{\mathrm{ij}}$ is transformed into a number $0 \sim 5$ respectively as shown in Table 2. In the same way, let $\mathrm{J}_{\mathrm{i}}$, (i.e., $\mathrm{R}$ and $\mathrm{P}$ joint) be 8 and 9 . Thus, the topological structure of a leg can be expressed as an integer matrix.

Table 2. Numbers of geometrical relationships of joints axes

Geometrical relationship Corresponding number




\begin{tabular}{cc}
\hline Geometrical relationship & Corresponding number \\
\hline parallel & 1 \\
orthogonal & 2 \\
coaxial & 3 \\
common point & 4 \\
coplanar & 5 \\
arbitrary & 0 \\
\hline
\end{tabular}

For example, the UP leg listed in Fig.2(a) has three pairs labeled with the numbers 8 , 8, and 9. The axes relationships are $R_{1} \perp R_{2}, R_{1} \perp P_{3}$, and $R_{2} \perp P_{3}$ respectively. Based on the above definition, the ordered topological matrix of this leg is

$$
\mathrm{L}=\left[\begin{array}{lll}
8 & 2 & 2 \\
2 & 8 & 2 \\
2 & 2 & 9
\end{array}\right]
$$

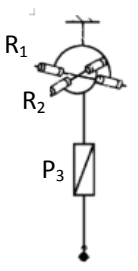

(a) UP

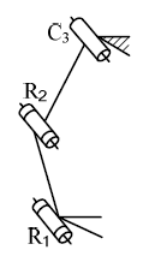

(b) RRC

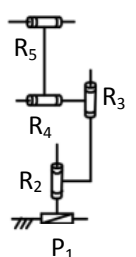

(c) PRRRR

Fig. 2. Typical legs not including $S$ pair

Generally, the multi-DOF kinematic pairs $C, U$ and $S$, can be equivalent to the combinations of $R_{1}{ }^{(C)} \mid P_{2}{ }^{(C)}$, $\mathrm{R}_{1}{ }^{(\mathrm{U})} \perp \mathrm{R}_{2}{ }^{(\mathrm{U})}$ and $\mathrm{R}_{1}{ }^{(\mathrm{S})}{ }_{*} \mathrm{R}_{2}{ }^{(\mathrm{S})} * \mathrm{R}_{3}{ }^{(\mathrm{S})}$ respectively. But the directions of the three equivalent axes of $\mathrm{S}$ pair should be calibrated according to the following criteria:

(1) When R pair and S pair are connected by a link, or connected by a prismatic pair or connected by a planar sub-chain, taking an equivalent $R^{(S)}$ pair parallel to the $R$ pair, as shown in Fig.3(a).

(2) When two S pairs are connected by a link, or connected by a prismatic pair, there is a local rotation DOF around the line connecting two points of the spherical centers. Then, one $S$ pair is treated as two $R$ pairs, and denoted as $\mathrm{R}_{1}{ }^{(\mathrm{S})}{ }_{*} \mathrm{R}_{2}{ }^{(\mathrm{S})}$. Meanwhile, its axes are calibrated according to the above criteria (1), as shown in Fig.3(b).

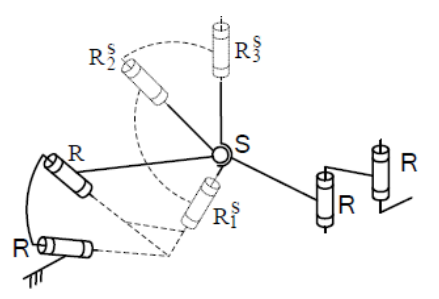

(a) RR-S-R ||R

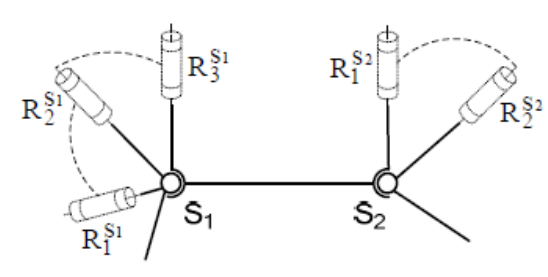

(b) $S_{1}-S_{2}$

Fig. 3. Equivalent axes of S pair

For example, the RPS leg listed in Fig.4(a) with $\mathrm{R}$ and $\mathrm{S}$ pairs, can be replaced with the topologically equivalent leg $R_{1}\left(\perp P_{2}\right)|| R_{3}-\left(R_{4}{ }^{*} R_{5}\right)$, as shown in Fig.4(b). Based on the above definition, the ordered topological matrix of this leg is 


$$
\mathrm{L}=\left[\begin{array}{lllll}
8 & 2 & 1 & 0 & 0 \\
2 & 9 & 2 & 0 & 0 \\
1 & 2 & 8 & 0 & 0 \\
0 & 0 & 0 & 8 & 4 \\
0 & 0 & 0 & 4 & 8
\end{array}\right]_{(\#)}
$$

where, the subscript symbol ' $\#$ ' indicates the serial number of the spherical center. Particularly, when different $S$ pairs (or three equivalent revolute pairs) have the same center, the serial number is the same.

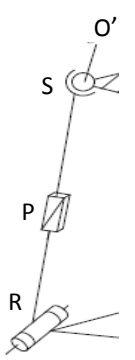

(a) RPS

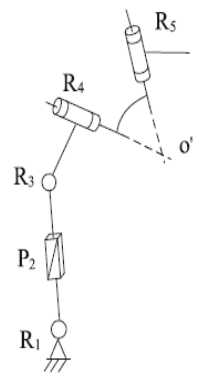

(b) RPRRR

Fig. 4. Typical legs including $S$ pair and its equivalent leg

\subsubsection{Definition of POC matrix of leg}

The POC matrix of a leg should include not only the dimension of output motion, but also the corresponding orientation of the motion. So, considering the relations between the topological structure and POC of a leg, a $2 \times f$ POC matrix $\left(M_{L}\right)$ is developed to match with its ordered topology matrix $L$, that is

$$
\mathrm{M}_{\mathrm{L}}=\left[\begin{array}{llll}
\mathrm{t}_{1} & \mathrm{~L} & \mathrm{t}_{\mathrm{i}} \mathrm{L} & \mathrm{t}_{\mathrm{f}} \\
\mathrm{r}_{1} & \mathrm{~L} & \mathrm{r}_{\mathrm{i}} \mathrm{L} & \mathrm{r}_{\mathrm{f}}
\end{array}\right]
$$

The defining of $M_{L}$ shall abide by the following criteria:

(1) $t_{i}\left(r_{i}\right)(i=1 \sim f)$ denotes translation (rotation) output respectively, and $t_{i}\left(r_{i}\right)=0,1,2$ or 3.

(2) $t_{i}\left(r_{i}\right) \neq 0$ indicates the existence of independent translation (rotation) output.

(3) when $t_{i}=3$ or $r_{i}=3$, the dimension of translation or rotation output is 3 . The directions are arbitrary in space and need not to be specified.

(4) The dimension of translation/rotation output is $\xi_{\mathrm{r}}=\sum_{\mathrm{i}=1}^{\mathrm{f}} \mathrm{r}_{\mathrm{i}} / \xi_{\mathrm{t}}=\sum_{\mathrm{i}=1}^{\mathrm{f}} \mathrm{t}_{\mathrm{i}}$, and the total dimension of output at the end link of the leg is $\xi=\xi_{\mathrm{r}}+\xi_{\mathrm{t}}$.

(5) Output direction of column $\mathrm{i}$ is related to the axis of the $\mathrm{i}^{\text {th }}$ joint in the ordered topological structure matrix L.

- $t_{i}=1$ means there is an independent translation along the direction of the $i^{\text {th }}$ joint axis (if $r_{i}=0$ ) or in the normal plane of the $i^{\text {th }}$ joint axis(if $r_{i}=1$ ).

- when $\mathrm{t}_{\mathrm{i}}=2$, there are two independent translations in the normal plane of the $\mathrm{i}^{\text {th }}$ joint axis.

Take the same UP leg as an example (Fig.2(a)). Its ordered topological structure matrix is Eq.(9), i.e. $\mathrm{L}=\left[\begin{array}{lll}8 & 2 & 2 \\ 2 & 8 & 2 \\ 2 & 2 & 9\end{array}\right]$. According to the definition, the POC can be expressed as $\mathrm{M}_{\mathrm{L}}=\left[\begin{array}{lll}0 & 0 & 1 \\ 1 & 1 & 0\end{array}\right]$. This matrix indicates as follows,

(1) The none-zero elements of $M_{L}$ mean the existing translation output (the first row) and the existing rotation output (the second row).

(2) The sum of the non-zero elements corresponds to the number of DOF of this leg. 
(3) Each column of $M_{L}$ implies the motion orientation, such as $r_{1}=1$ denotes that the rotation direction corresponds to the axis of the $1^{\text {st }}$ pair (i.e. $R_{1}$ joint) in the leg. Similarly, $r_{2}=1$ corresponds to the $R_{2}$ joint and $t_{3}=1$ (with $r_{3}=0$ ) corresponds to the translation direction along the $P_{3}$ joint axis.

\subsubsection{POC matrix of a sub-chain}

More commonly, planar sub-chains (2-DOF $\mathrm{G}_{2}$ and 3-DOF $\mathrm{G}_{3}$ ) or spherical sub-chains (2-DOF $\mathrm{S}_{2}$ and 3-DOF $\mathrm{S}_{3}$ ) may constitute a leg or part of a leg consisting of parallel or orthogonal $R$ joints and/or $P$ joints. It is not difficult to see that, no matter which point on the end link is chosen, the end link of a planar sub-chain has always a onedimensional rotation and a one-dimensional or two-dimensional independent translations in the normal plane of the $R$ joint axis. On the contrary, for a spherical sub-chain (2-DOF $S_{2}$ and 3-DOF $S_{3}$ ), different points of an end link have different characteristics of POC.

In order to express the motion output direction more clearly and facilitate subsequent operations of POCs, it is necessary to extract the sub-chains and to mention the specific point of the end-effectors. In accordance with the definition of a POC matrix, 9 type matrices for the 12 sub-chains is illustrated in Table 3, where POC of the subchains $R_{1} * R_{2}$ and $R_{1}{ }^{*} R_{2} * R_{3}$ have two different forms. Here, we take the sub-chain $R_{1} * R_{2}{ }^{*} R_{3}$ as an example to illustrate what the two matrices mean and how to choose in the calculation. The specific description is given as follows

(1) If the intersection point of three R joints' axes is selected as base point $O^{\prime}$, there exist three independent finite rotations parallel to the axes of $R_{1}, R_{2}$ and $R_{3}$ respectively. So the $P O C$ is written as $\left[\begin{array}{ccc}0 & 0 & 0 \\ 1 & 1 & 1\end{array}\right]$.

(2) If base point $O^{\prime}$ is selected outside the axis of the end joint $\left(R_{3}\right)$, there are also two derivative finite translations within a plane perpendicular to radius vector $\rho$ shown in Fig.5. Therefore, in addition to the three finite rotations mentioned above, two non-independent translations should be considered (See detailed proof in [18]). So the POC can be expressed as $\left[\begin{array}{lll}0 & 0 & 0 \\ 1 & 1 & 1\end{array}\right]+\left[\begin{array}{l}2 \\ \#\end{array}\right]$, where the symbol '\#' denotes the No. of intersection point, and the number 2 indicates the two-dimensional derivative translations, which is non-independent.

Table 3 Twelve Sub-chains and their POCs

\begin{tabular}{|c|c|c|c|c|c|}
\hline No. & Type & Symbol & Dimension & Topology Matrix & POC matrix \\
\hline 1 & & $R|| R$ & 2 & {$\left[\begin{array}{ll}8 & 1 \\
1 & 8\end{array}\right]$} & {$\left[\begin{array}{ll}1 & 0 \\
1 & 0\end{array}\right]$} \\
\hline 2 & $\mathrm{G}_{2}$ & $\mathrm{R} \perp \mathrm{P}$ & 2 & {$\left[\begin{array}{ll}8 & 2 \\
2 & 9\end{array}\right]$} & {$\left[\begin{array}{ll}1 & 0 \\
1 & 0\end{array}\right]$} \\
\hline 3 & & $\mathrm{P} \perp \mathrm{R}$ & 2 & {$\left[\begin{array}{ll}9 & 2 \\
2 & 8\end{array}\right]$} & {$\left[\begin{array}{ll}0 & 1 \\
0 & 1\end{array}\right]$} \\
\hline 4 & & $\mathrm{R} / / \mathrm{R} / / \mathrm{R}$ & 3 & {$\left[\begin{array}{lll}8 & 1 & 1 \\
1 & 8 & 1 \\
1 & 1 & 8\end{array}\right]$} & {$\left[\begin{array}{lll}2 & 0 & 0 \\
1 & 0 & 0\end{array}\right]$} \\
\hline 5 & & $\mathrm{R} / / \mathrm{R} \perp \mathrm{P}$ & 3 & {$\left[\begin{array}{lll}8 & 1 & 2 \\
1 & 8 & 2 \\
2 & 2 & 9\end{array}\right]$} & {$\left[\begin{array}{lll}2 & 0 & 0 \\
1 & 0 & 0\end{array}\right]$} \\
\hline 6 & $\mathrm{G}_{3}$ & $P \perp R / / R$ & 3 & {$\left[\begin{array}{lll}9 & 2 & 2 \\
2 & 8 & 1 \\
2 & 1 & 8\end{array}\right]$} & {$\left[\begin{array}{lll}0 & 2 & 0 \\
0 & 1 & 0\end{array}\right]$} \\
\hline 7 & & $\mathrm{R}(\perp \mathrm{P}) / / \mathrm{R}$ & 3 & {$\left[\begin{array}{lll}8 & 2 & 1 \\
2 & 9 & 2 \\
1 & 2 & 8\end{array}\right]$} & {$\left[\begin{array}{lll}2 & 0 & 0 \\
1 & 0 & 0\end{array}\right]$} \\
\hline 8 & & $\mathrm{R}(\perp \mathrm{P}) \perp \mathrm{P}$ & 3 & {$\left[\begin{array}{lll}8 & 2 & 2 \\
2 & 9 & 2 \\
2 & 2 & 9\end{array}\right]$} & {$\left[\begin{array}{lll}2 & 0 & 0 \\
1 & 0 & 0\end{array}\right]$} \\
\hline
\end{tabular}




\begin{tabular}{|c|c|c|c|c|c|}
\hline No. & Type & Symbol & Dimension & Topology Matrix & POC matrix \\
\hline 9 & & $\mathrm{P}(\perp \mathrm{P}) \perp \mathrm{R}$ & 3 & {$\left[\begin{array}{lll}9 & 2 & 2 \\
2 & 9 & 2 \\
2 & 2 & 8\end{array}\right]$} & {$\left[\begin{array}{lll}0 & 0 & 2 \\
0 & 0 & 1\end{array}\right]$} \\
\hline 10 & & $P(\perp R) \perp P$ & 3 & {$\left[\begin{array}{lll}9 & 2 & 2 \\
2 & 8 & 2 \\
2 & 2 & 9\end{array}\right]$} & {$\left[\begin{array}{lll}0 & 2 & 0 \\
0 & 1 & 0\end{array}\right]$} \\
\hline 11 & $\mathrm{~S}_{2}$ & $\mathrm{R}^{*} \mathrm{R}$ & 2 & {$\left[\begin{array}{ll}8 & 4 \\
4 & 8\end{array}\right]$} & $\begin{array}{c}{\left[\begin{array}{ll}0 & 0 \\
1 & 1\end{array}\right]} \\
{\left[\begin{array}{ll}0 & 0 \\
1 & 1\end{array}\right]+\left[\begin{array}{l}2 \\
\#\end{array}\right]}\end{array}$ \\
\hline 12 & $\mathrm{~S}_{3}$ & $\begin{array}{c}\mathrm{R}_{1} * \mathrm{R}_{2} * \mathrm{R}_{3} \\
\text { (Not coplanar) }\end{array}$ & 3 & {$\left[\begin{array}{lll}8 & 4 & 4 \\
4 & 8 & 4 \\
4 & 4 & 8\end{array}\right]$} & $\begin{array}{c}{\left[\begin{array}{lll}0 & 0 & 0 \\
1 & 1 & 1\end{array}\right]} \\
{\left[\begin{array}{lll}0 & 0 & 0 \\
1 & 1 & 1\end{array}\right]+\left[\begin{array}{l}2 \\
\#\end{array}\right]}\end{array}$ \\
\hline & & & & & \\
\hline
\end{tabular}

Fig. 5. The sub-chain of $R_{1} * R_{2} * R_{3}$

\subsubsection{Topology matrix and POC matrix of some typical legs}

Table 4 displays seven typical legs and their topological matrices and the corresponding POC matrices, among which the structures of legs No.3 No.6 are shown in Fig.2(a)-(c) and Fig.4(a). Clearly, the representation of the topological structure and the POC of a leg exhibits an orderly one-to-one correspondence among the sequence of the pairs and the motion output orientations.

Table 4. Topology matrix and POC matrix of typical legs

\begin{tabular}{|c|c|c|c|c|c|}
\hline No. & Symbol & Topology Matrix & POC matrix & & Commentary \\
\hline 1 & $\mathrm{R}$ & $L=[8]$ & $\mathrm{M}_{\mathrm{L}}=\left[\begin{array}{l}0 \\
1\end{array}\right]$ & & Rotation around $\mathrm{R}$ axis \\
\hline 2 & $\mathrm{P}$ & $\mathrm{L}=[9]$ & $\mathrm{M}_{\mathrm{L}}=\left[\begin{array}{l}1 \\
0\end{array}\right]$ & & Translation along $\mathrm{P}$ axis \\
\hline 3 & $\begin{array}{c}\text { UP } \\
\left(R_{1} R_{2} P_{3}\right)\end{array}$ & $\mathrm{L}=\left[\begin{array}{lll}8 & 2 & 2 \\
2 & 8 & 2 \\
2 & 2 & 9\end{array}\right]$ & $\mathrm{M}_{\mathrm{L}}=\left[\begin{array}{lll}0 & 0 & 1 \\
1 & 1 & 0\end{array}\right]$ & & $\begin{array}{l}\text { - One-translation along } P_{3} \text { axis } \\
\text { - Two-rotations about axes of } R_{1} \text { and } R_{2} \text { joints }\end{array}$ \\
\hline 4 & $\begin{array}{c}\mathrm{RRC} \\
\left(\mathrm{R}_{1} \mathrm{R}_{2} \mathrm{R}_{3} \mathrm{P}_{4}\right)\end{array}$ & $\mathrm{L}=\left[\begin{array}{llll}8 & 1 & 1 & 1 \\
1 & 8 & 1 & 1 \\
1 & 1 & 8 & 1 \\
1 & 1 & 1 & 9\end{array}\right]$ & $\mathrm{M}_{\mathrm{L}}=\left[\begin{array}{ll}3 & 0 \\
1 & 0\end{array}\right.$ & $\left.\begin{array}{ll}0 & 0 \\
0 & 0\end{array}\right]$ & $\begin{array}{l}\text { - Three-translations along arbitrary directions in } \\
\text { space } \\
\text { - One-rotation about } \mathrm{R}_{1} \text { joint axis }\end{array}$ \\
\hline 5 & $P_{1} R_{2} R_{3} R_{4} R_{5}$ & $\mathrm{~L}=\left[\begin{array}{lllll}9 & 2 & 2 & 1 & 1 \\
2 & 8 & 1 & 2 & 2 \\
2 & 1 & 8 & 2 & 2 \\
1 & 2 & 2 & 8 & 1 \\
1 & 2 & 2 & 1 & 8\end{array}\right]$ & $\mathrm{M}_{\mathrm{L}}=\left[\begin{array}{ll}3 & 0 \\
0 & 1\end{array}\right.$ & $\left.\begin{array}{lll}0 & 0 & 0 \\
0 & 1 & 0\end{array}\right]$ & $\begin{array}{l}\text { - Three-translations along arbitrary directions in } \\
\text { space } \\
\text { - Two-rotations about } \mathrm{R}_{2} \text { and } \mathrm{R}_{4} \text { joints axes }\end{array}$ \\
\hline
\end{tabular}




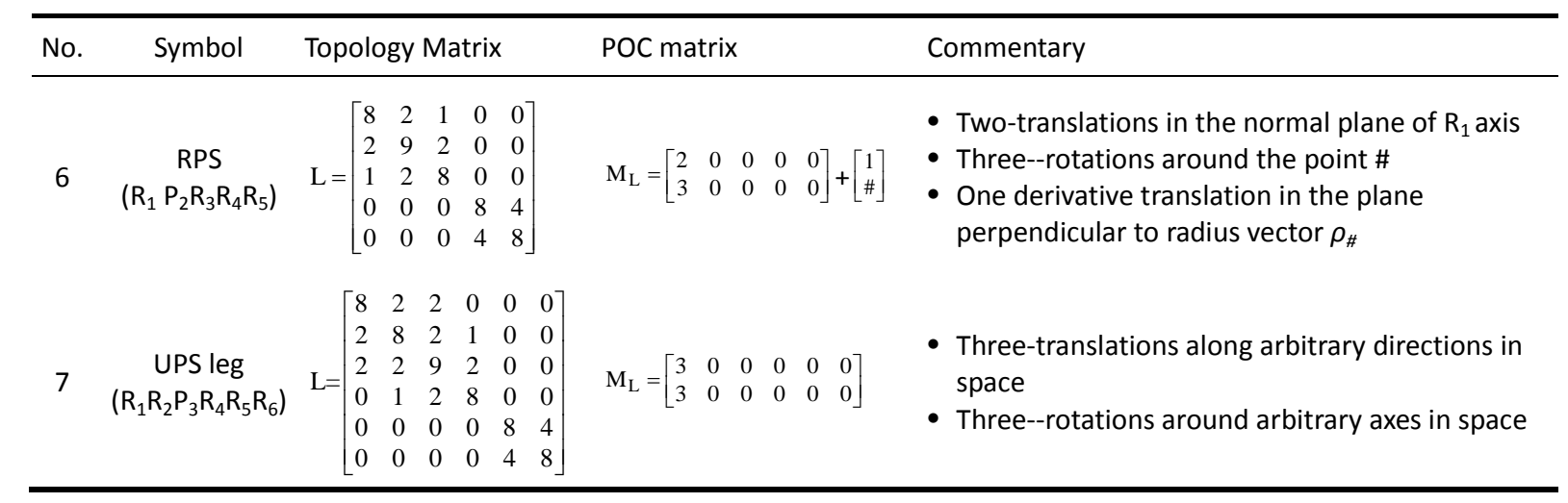

\subsection{Description of a PM and its POC}

As mentioned previously, the mobility of a PM depends not only on the geometrical axes relationships in one leg, but also on the geometrical axes relationships between legs. Therefore, in addition to the topological structures of all legs, the topological relations between the legs of a PM are needed to be considered in digital form for the mobility analysis.

In most cases, the geometrical axes relationships between legs depend on the invariant geometric relations between joints axes respectively on the moving platform and on the fixed platform.

To make information concise, the representation of PMs is developed to clearly represent the composition of legs and the relationships of axes pairs on the two platforms.

\subsubsection{Description of PM}

The representation of the topological structure for a given PM with $\mathrm{n}$ legs is expressed in the following four steps.

Step 1 Label all $n$ legs comprising the PM in turn with numbers $1 \sim n$.

Step 2 Obtain the ordered topological matrix $L_{i}(i=1 \sim n)$ of all $n$ legs.

Step 3 Definite two virtual legs MP and BP constituted by the pairs on the moving platform and on the fixed platform in the order of legs.

Step 4 Obtain the ordered topological matrix $L_{M P}$ and $L_{B P}$ of the virtual legs MP and $B P$.

Then, the topological composition of the PM can be expressed as

$$
P M=\left(L_{1}, L_{2}, \ldots, L_{n}, L_{M P}, L_{B P}\right)
$$

It should be pointed out that if a leg is connected to the fixed platform or to the moving platform by a P pair of a planar sub-chain, the normal of the sub-chain is represented rather than the axis of the $P$ pair. This is because the output direction of planar sub-chain is represented by the axis of the $R$ pair which is perpendicular to the $P$ pair ( See No. 3, 6, 9 and 10 in Table 3).

Three examples are given here to illustrate how the proposed representation works.

(1) 3-UPS \& 1-UP PM

The PM in Fig.6(a) has 3-UPS legs and 1-UP leg. First, replace composite pairs $S$ and $U$ with three $R$ pairs with concurrent axes and two $R$ pairs with orthogonal axes respectively. Then, label the legs as shown in Fig.6(a). In what follows, obtain the two virtual legs MP and BP as R-R-R-R and R-R-R-P, and indicate that the axes relationships of all joints on the two platforms are arbitrary.

Accordingly, the topological composition of this $P M$ is $P M=\left(L_{1}, L_{2}, L_{3}, L_{4}, L_{M P}, L_{B P}\right)$.

The topology matrix $L_{i}(i=1 \sim 3)$ for 3-UPS legs and the matrix $L_{4}$ for the UP leg are expressed as follows.

$$
\mathrm{L}_{\mathrm{i}}=\left[\begin{array}{llllll}
8 & 2 & 2 & 0 & 0 & 0 \\
2 & 8 & 2 & 1 & 0 & 0 \\
2 & 2 & 9 & 2 & 0 & 0 \\
0 & 1 & 2 & 8 & 0 & 0 \\
0 & 0 & 0 & 0 & 8 & 4 \\
0 & 0 & 0 & 0 & 4 & 8
\end{array}\right] \quad(\mathrm{i}=1 \sim 3), \quad \mathrm{L}_{4}=\left[\begin{array}{lll}
8 & 2 & 2 \\
2 & 8 & 2 \\
2 & 2 & 9
\end{array}\right]
$$


Matrix $L_{M P}$ and $L_{B P}$ are

$$
\mathrm{L}_{\mathrm{MP}}=\left[\begin{array}{llll}
8 & 0 & 0 & 0 \\
0 & 8 & 0 & 0 \\
0 & 0 & 8 & 0 \\
0 & 0 & 0 & 9
\end{array}\right], \quad \mathrm{L}_{\mathrm{BP}}=\left[\begin{array}{llll}
8 & 0 & 0 & 0 \\
0 & 8 & 0 & 0 \\
0 & 0 & 8 & 0 \\
0 & 0 & 0 & 8
\end{array}\right]
$$

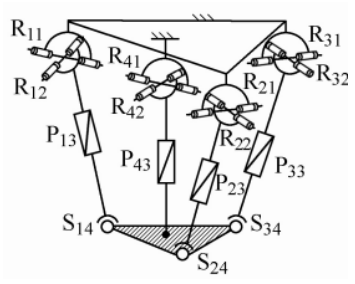

(a) 3-UPS\&1-UP

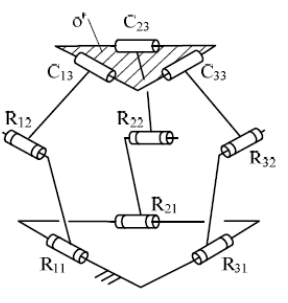

(b) 3-RRC

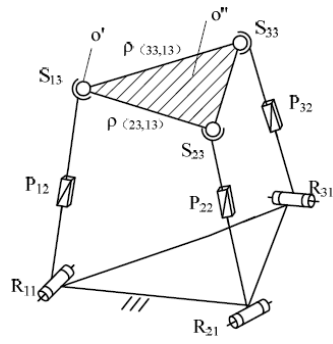

(c) 3-RPS

Fig.6. Four PMs

(2) 3-RRC PM

The 3-RRC PM in Fig.6(b) has three identical RRC legs. First, replace composite pairs $C$ with one $R$ joint and one $P$ joint with collinear axes. Then, label the legs as shown in Fig.6(b) and obtain the two virtual legs MP and BP as P$\mathrm{P}-\mathrm{P}$ and $\mathrm{R}-\mathrm{R}-\mathrm{R}$, and the axes relationships of all joints on the two platforms are coplanar.

Accordingly, the topological composition of 3-RRC $P M$ is $P M=\left(L_{1}, L_{2}, L_{3}, L_{M P}, L_{B P}\right)$.

The topological structure matrices $L_{i}(i=1 \sim 3)$ for 3-RRC legs are

$$
\mathrm{L}_{\mathrm{i}}=\left[\begin{array}{llll}
8 & 1 & 1 & 1 \\
1 & 8 & 1 & 1 \\
1 & 1 & 8 & 1 \\
1 & 1 & 1 & 9
\end{array}\right] \quad(\mathrm{i}=1 \sim 3)
$$

Matrix $L_{M P}$ and $L_{B P}$ are

$$
\mathrm{L}_{\mathrm{MP}}=\left[\begin{array}{lll}
9 & 5 & 5 \\
5 & 9 & 5 \\
5 & 5 & 9
\end{array}\right] \text { and } \mathrm{L}_{\mathrm{BP}}=\left[\begin{array}{lll}
8 & 5 & 5 \\
5 & 8 & 5 \\
5 & 5 & 8
\end{array}\right]
$$

(3) 3-RPS PM

The 3-RPS PM in Fig.6(c) has three identical RPS legs. First, replace composite pairs $S$ with one R joint and two intersecting $R$ joints. Then, label the legs as shown in Fig.6(c) and obtain the two virtual legs MP and BP both as R-R$\mathrm{R}$, and the axes relationships of all joints on the two platforms are arbitrary.

Thus, the topological composition of 3-RPS PM is $P M=\left(L_{1}, L_{2}, L_{3}, L_{M P}, L_{B P}\right)$.

The topological structure matrices $L_{i}(i=1 \sim 3)$ for 3-RPS legs are

$$
\mathrm{L}_{\mathrm{i}}=\left[\begin{array}{lllll}
8 & 2 & 1 & 0 & 0 \\
2 & 9 & 2 & 0 & 0 \\
1 & 2 & 8 & 2 & 2 \\
0 & 0 & 2 & 8 & 4 \\
0 & 0 & 2 & 4 & 8
\end{array}\right]_{(\mathrm{i})} \quad(\mathrm{i}=1 \sim 3)
$$

where, the subscript i represents the No. of intersection point of the last two revolute joints axes.

Matrix $L_{M P}$ and $L_{B P}$ are

$$
\mathrm{L}_{\mathrm{MP}}=\left[\begin{array}{lll}
8 & 0 & 0 \\
0 & 8 & 0 \\
0 & 0 & 8
\end{array}\right] \text { and } \mathrm{L}_{\mathrm{BP}}=\left[\begin{array}{lll}
8 & 0 & 0 \\
0 & 8 & 0 \\
0 & 0 & 8
\end{array}\right]
$$

\subsubsection{Description of POC of a PM}


According to the intersection property of sets, the direction of translation/rotation output of a PM is related to one or more joints in one of the legs. Thus, the number of this leg should be recorded for reference. So, the POC of a PM can be completely expressed by adding the number of reference to the legs on the basis of POC of a serial leg. It is defined as

$$
M_{p a}=\left[\begin{array}{llll}
t_{1} & L & t_{i} L & t_{n j} \\
r_{1} & L & r_{i} L & r_{n j}
\end{array}\right]\left[L_{j}\right],\left(n_{j} \leq 6\right)
$$

where, $L_{j}$ is the number of the reference leg specifying the direction of translation/rotation output, $n_{j}$ is the number of 1-DOF pairs in leg $\mathrm{L}_{\mathrm{j}}$.

We show this representation with three PMs shown in Fig.6. The PM shown in Fig.6(a) has 2-rotational outputs and 1-translational output, and the POC matrix of this PM can be expressed as $M_{p a}=\left[\begin{array}{lll}0 & 0 & 1 \\ 1 & 1 & 0\end{array}\right]\left[\begin{array}{ll}4\end{array}\right]$. The right element " 4 " indicates that the $4^{\text {th }}$ leg (UP leg) is the reference leg for the output directions of the moving platform, which means that this mechanism has a translation output along the $\mathrm{P}_{43}$ joint axis, and the directions of rotation outputs are around $\mathrm{R}_{41}$ and $\mathrm{R}_{42}$ joint axes.

The 3-RRC PM shown in Fig.6(b) has 3-translations and its POC is $M_{p a}=\left[\begin{array}{lll}3 & 0 & 0 \\ 0 & 0 & 0\end{array}\right][1]$, which shows that 3-RRC has a 3-dimensional translation output and the element ' 1 ' indicates the $1^{\text {st }}$ leg is the output reference. In fact, 3 dimensional translation output can translate along arbitrary space direction and needs not to be specified.

For the PM in Fig.6(c), point $\mathrm{O}^{\prime}$ on the moving platform is selected as the base point, and its $\mathrm{POC}$ is $\mathrm{M}_{\mathrm{pa}}=\left[\begin{array}{lll}2 & 0 & 0 \\ 3 & 0 & 0\end{array}\right][1]$, which shows that 3-RPS has a 3-dimensional rotation outputs and 2-dimensional translation outputs perpendicular to the axis $\mathrm{R}_{11}$. In fact, since DOF of the PM is three, the POC has just three independent elements, and the other two elements are non-independent.

\section{Automatic analysis of POC and dimension of independent displacement equations}

\subsection{Automatic POC analysis of a leg}

\subsubsection{Algorithm principle}

Based on Eq.(3), the POC of a leg is the union operation on POCs of all joints.

Each leg can be considered as a combination of a series of independent constituent units, such as plane subchains $\left(G_{3}\right.$ and $\left.G_{2}\right)$, spherical sub-chains $\left(S_{3}\right.$ and $\left.S_{2}\right)$ and the remaining 1-DOF $R$ and $P$ joints. Each component unit has a unique corresponding POC matrix (Listed in Table 3 and Table 4). Thus, according to the topological structure matrix defined above, calculating the POC of a leg can be mapped into simply combining the POC matrices of these independent constituent units.

Table 3 and Table 4 show that the POC matrices of these independent constituent units have different dimensions. For convenience of programming, $\mathrm{POC}$ matrices of these units (named as $M_{\mathrm{G} 3}, \mathrm{M}_{\mathrm{G} 2}, \mathrm{M}_{\mathrm{S} 3}, \mathrm{M}_{\mathrm{S} 2}$ and $\mathrm{M}_{\mathrm{J}}$ ) will be supplemented to a $2 \times 6$ matrix to be dimensionally consistent.

$$
M_{*(s p)}=\left[\begin{array}{llllll}
t_{1} & t_{2} & t_{3} & t_{4} & t_{5} & t_{6} \\
r_{1} & r_{2} & r_{3} & r_{4} & r_{5} & r_{6}
\end{array}\right]
$$

The symbol ' $*$ ' is to be used to represent $\mathrm{G}_{3}, \mathrm{G}_{2}, \mathrm{~S}_{3}, \mathrm{~S}_{2}$ or J.

The specific complement rule must preserve the order of the constituent units in the leg. Take a $P_{1} R_{2} R_{3} R_{4} R_{5}$ leg shown in Fig.2(c) as an example.

In Fig.2(c), the two serial sub-chains $G_{3}\left(P_{1} \perp R_{2} / / R_{3}\right)$ and $G_{2}\left(R_{4} / / R_{5}\right)$ can be extracted. Their original $P O C$ matrices and the supplemented POC matrices are as follows: 


$$
\begin{aligned}
M_{\mathrm{G} 3}=\left[\begin{array}{lll}
0 & 2 & 0 \\
0 & 1 & 0
\end{array}\right] & \Rightarrow \mathrm{M}_{\mathrm{G} 3(\mathrm{sp})}=\left[\begin{array}{llllll}
0 & 2 & 0 & 0 & 0 & 0 \\
0 & 1 & 0 & 0 & 0 & 0
\end{array}\right] \\
\mathrm{M}_{\mathrm{G} 2}=\left[\begin{array}{ll}
1 & 0 \\
1 & 0
\end{array}\right] & \Rightarrow M_{\mathrm{G} 2(\mathrm{sp})}=\left[\begin{array}{llllll}
0 & 0 & 0 & 1 & 0 & 0 \\
0 & 0 & 0 & 1 & 0
\end{array}\right]
\end{aligned}
$$

Similarly, for the leg of RPS in Fig.4(a), sub-chains $G_{3}\left(R_{1} \perp P_{2} / / R_{3}\right)$ and $S_{2}\left(R_{4}{ }^{*} R_{5}\right)$ can be extracted. If point $O^{\prime}$ is selected as the base point, their original POC matrices and the supplemented POC matrices are described by

$$
\begin{aligned}
& \mathrm{M}_{\mathrm{G} 3}=\left[\begin{array}{lll}
2 & 0 & 0 \\
1 & 0 & 0
\end{array}\right] \Rightarrow \mathrm{M}_{\mathrm{G} 3(\mathrm{sp})}=\left[\begin{array}{llllll}
2 & 0 & 0 & 0 & 0 & 0 \\
1 & 0 & 0 & 0 & 0 & 0
\end{array}\right] \\
& \mathrm{M}_{\mathrm{S} 2}=\left[\begin{array}{lll}
0 & 0 & 0 \\
1 & 1 & 0
\end{array}\right] \Rightarrow \mathrm{M}_{\mathrm{S} 2(\mathrm{sp})}=\left[\begin{array}{llllll}
0 & 0 & 0 & 0 & 0 & 0 \\
0 & 0 & 0 & 1 & 1 & 0
\end{array}\right]
\end{aligned}
$$

It can be seen that the sequence of joints is retained while expressing the POC.

Thus, the POC of a leg can be calculated as

$$
\mathrm{M}_{\mathrm{L}(\mathrm{sp})}=\mathrm{M}_{\mathrm{G}_{3}(\mathrm{sp})}\left\|\mathrm{M}_{\mathrm{G}_{2}(\mathrm{sp})}\right\| \mathrm{M}_{\mathrm{S}_{3}(\mathrm{sp})}\left\|\mathrm{M}_{\mathrm{S}_{2}(\mathrm{sp})}\right\| \mathrm{M}_{\mathrm{J}(\mathrm{sp})}
$$

where $M_{G 3(s p)}, M_{G 2(s p)}, M_{S 3(s p)}, M_{S 2(s p)}$ and $M_{J(s p)}$ are the supplemented forms of the POC matrices of $G_{3}, G_{2}, S_{3}, S_{2}$ and 1-DOF joints. The symbol 'II' denotes the logical 'or' operation in each bit of the matrices. As long as one corresponding bit is 1 , the result is ' 1 '; otherwise, is ' 0 '.

Hence, one can reason that the process of $\mathrm{POC}$ analysis of a leg will be converted into the process of extracting sub-chains and supplementing POC matrices.

\subsubsection{Analysis algorithm procedure for the POC of a leg}

Here, based on the topology matrices of legs and the extracted sub-chains and 1-DOF joints, an automatic POC analysis method of a leg is presented.

Step 1 Determine the topology matrix of the leg, and extract successively $G_{3}, G_{2}, S_{3}, S_{2}$ and the remaining 1DOF joints.

Step 2 Select base point O'.

Step 3 Determine the supplemented matrix of the extracted $G_{3}, G_{2}, S_{3}, S_{2}$ and the matrix of remaining 1-DOF joints.

Step 4 Carry out the bitwise "or" operation on the supplemented matrices of $G_{3}, G_{2}, S_{3}, S_{2}$ and 1-DOF joints, and obtain the initial POC matrix, which is denoted as $\mathrm{M}_{\mathrm{L}}^{0}=\left[\begin{array}{llllll}\mathrm{t}_{1} & \mathrm{t}_{2} & \mathrm{t}_{3} & \mathrm{t}_{4} & \mathrm{t}_{5} & \mathrm{t}_{6} \\ \mathrm{r}_{1} & \mathrm{r}_{2} & \mathrm{r}_{3} & \mathrm{r}_{4} & \mathrm{r}_{5} & \mathrm{r}_{6}\end{array}\right], \sum_{\mathrm{i}=1}^{6} \mathrm{r}_{\mathrm{i}}=\xi_{\mathrm{r}}^{0}, \sum_{\mathrm{i}=1}^{6} \mathrm{t}_{\mathrm{i}}=\xi_{\mathrm{t}}^{0}$.

Step 5 Generate the final POC matrix by converting redundant parallel revolute output into translation output according to the following three rules. The result is recorded as $\mathrm{M}_{\mathrm{L}}=\left[\begin{array}{llllll}\mathrm{t}_{1} & \mathrm{t}_{2} & \mathrm{t}_{3} & \mathrm{t}_{4} & \mathrm{t}_{5} & \mathrm{t}_{6} \\ \mathrm{r}_{1} & \mathrm{r}_{2} & \mathrm{r}_{3} & \mathrm{r}_{4} & \mathrm{r}_{5} & \mathrm{r}_{6}\end{array}\right], \sum_{\mathrm{i}=1}^{6} \mathrm{r}_{\mathrm{i}}=\xi_{\mathrm{r}}, \sum_{\mathrm{i}=1}^{6} \mathrm{t}_{\mathrm{i}}=\xi_{\mathrm{t}}$.

- If $\xi_{r}^{0}>3$, then correct $\xi_{r}=3$ and $\xi_{t}=\xi_{t}^{0}+\xi_{r}^{0}-3$, and the translation direction newly augmented lies in the normal plane of the parallel revolute joints.

- If $\xi_{r}^{0} \leq 3$, the final POC matrix is $M_{L}=M_{L}^{0}$, i.e., $\xi_{t}=\xi_{t}^{0}$ and $\xi_{r}=\xi_{r}^{0}$.

- If $\xi_{\mathrm{t}}=2$ (or 3 ), and the leg exists a two-dimensional derivative translations, then the two-dimensional translations will be reduced to one (or zero) dimension.

\subsubsection{Examples of POC analysis of a leg}

Example 1 Determine the $P O C$ of the leg $P \perp R / / R \perp R / / R$, which is shown in Fig.2(c).

Step 1 Recognize and sequentially extract sub-chains. There is one $G_{3}$ sub-chain $P \perp R / / R$ and one $G_{2}$ planar sub-chain $\mathrm{R} / / \mathrm{R}$.

Step 2 Select base point O' outside the axis of the end R pair. 
Step 3 Generate the supplemented POC matrices of $\mathrm{G}_{3}$ and $\mathrm{G}_{2}$ as $\mathrm{M}_{\mathrm{G} 3}=\left[\begin{array}{llllll}0 & 2 & 0 & 0 & 0 & 0 \\ 0 & 1 & 0 & 0 & 0 & 0\end{array}\right]$, $\mathrm{M}_{\mathrm{G} 2}=\left[\begin{array}{llllll}0 & 0 & 0 & 1 & 0 & 0 \\ 0 & 0 & 0 & 1 & 0 & 0\end{array}\right]$

Step 4 Carry out bitwise "or" operation on $M_{G 3}$ and $M_{G 2}$, thus the initial POC matrix is $\mathrm{M}_{\mathrm{L}}^{0}=\left[\begin{array}{cccccc}0 \mathrm{P} 0 & 2 \mathrm{P} 0 & 0 \mathrm{P} 0 & 0 \mathrm{P} 1 & 0 \mathrm{P} 0 & 0 \mathrm{P} 0 \\ 0 \mathrm{P} 0 & 1 \mathrm{P} 0 & 0 \mathrm{P} 0 & 0 \mathrm{P} 1 & 0 \mathrm{P} 0 & 0 \mathrm{P} 0\end{array}\right]=\left[\begin{array}{llllll}0 & 2 & 0 & 1 & 0 & 0 \\ 0 & 1 & 0 & 1 & 0 & 0\end{array}\right]$, and $\xi_{\mathrm{r}}^{0}=2$, and $\xi_{\mathrm{t}}^{0}=3$.

Step $5 \xi_{t}^{0}=3$ and $\xi_{r}^{0}=2$, so $M_{L}=M_{L}^{0}$, that means $M_{L}=\left[\begin{array}{llllll}3 & 0 & 0 & 0 & 0 & 0 \\ 0 & 1 & 0 & 1 & 0 & 0\end{array}\right]$, and $\xi_{t}=3$ indicates that the leg has three independent translation outputs along arbitrary space directions, $\xi_{r}=2$ indicates that it has two rotational outputs, $r_{2}=r_{4}=1$ means the two rotations axes are around the joints of $R_{2}$ and $R_{4}$, respectively.

Example 2 Determine the POC of the RPS leg shown in Fig.4(a).

Step 1 Recognize and sequentially extract sub-chains. There is one $G_{3}$ sub-chain $R \perp P / / R$ and one $S_{2}$ sub-chain $\mathrm{R}^{*} \mathrm{R}$.

Step 2 Select base point $O^{\prime}$ outside the axis of the end R pair.

Step 3 Generate the supplemented POC matrices of $\mathrm{G}_{3}$ and $\mathrm{G}_{2}$ as $\mathrm{M}_{\mathrm{G} 3}=\left[\begin{array}{llllll}2 & 0 & 0 & 0 & 0 & 0 \\ 1 & 0 & 0 & 0 & 0 & 0\end{array}\right]$, $\mathrm{M}_{\mathrm{S} 2}=\left[\begin{array}{llllll}0 & 0 & 0 & 0 & 0 & 0 \\ 0 & 0 & 0 & 1 & 1 & 0\end{array}\right]+\left[\begin{array}{c}2 \\ \# 1\end{array}\right]$.

Step 4 Carry out bitwise "or" operation on $M_{G 3}$ and $M_{G 2}$, thus the initial POC matrix is $\mathrm{M}_{\mathrm{L}}{ }^{0}=\left[\begin{array}{llllll}2 \mathrm{P} 0 & 0 \mathrm{P} 0 & 0 \mathrm{P} 0 & 0 \mathrm{P} 0 & 0 \mathrm{P} 0 & 0 \mathrm{P} 0 \\ 1 \mathrm{P} 0 & 0 \mathrm{P} 0 & 0 \mathrm{P} 0 & 0 \mathrm{P} 1 & 0 \mathrm{P} 1 & 0 \mathrm{P} 0\end{array}\right]+\left[\begin{array}{c}2 \\ \# 1\end{array}\right]=\left[\begin{array}{llllll}2 & 0 & 0 & 0 & 0 & 0 \\ 3 & 0 & 0 & 0 & 0 & 0\end{array}\right]+\left[\begin{array}{c}2 \\ \# 1\end{array}\right]$, and $\xi_{\mathrm{r}}^{0}=3$, and $\xi_{\mathrm{t}}^{0}=2, \quad\left[\begin{array}{c}2 \\ \# 1\end{array}\right]$.

Step $5 \xi_{r}^{0}=3, \xi_{t}^{0}=2$ and $\left[\begin{array}{c}2 \\ \# 1\end{array}\right]$, so $M_{L}=\left[\begin{array}{llllll}2 & 0 & 0 & 0 & 0 & 0 \\ 3 & 0 & 0 & 0 & 0 & 0\end{array}\right]+\left[\begin{array}{c}1 \\ \# 1\end{array}\right]$. Therefore, the leg has two independent translational outputs in the normal plane of axis $R_{1}$ and three rotational outputs around the spherical center \#1, and $\left[\begin{array}{c}1 \\ \# 1\end{array}\right]$ shows that the effector has one derivative translation within the plane perpendicular to radius vector $\rho_{1}$.

\subsection{Automatic POC analysis of a PM}

POC of a PM is the result of the intersection operations on all legs in the PM. Among them, the translation output of the PM is the intersection of translational outputs of all legs, and the same works for the rotation outputs.

According to the kinematic characteristics of rigid body, the rotational outputs has no effect on the translational output, but the translational outputs affect the position of the rotation axis. So intersection rules for rotation outputs of POC will be more complicated.

Given are two legs $L_{1}$ and $L_{2}$, numbered as $I_{1}$ and $I_{2}$ respectively. The POC matrices of these legs are described as $\mathrm{M}_{\mathrm{Li}}=\left[\begin{array}{llllll}\mathrm{t}_{\mathrm{i} 1} & \mathrm{t}_{\mathrm{i} 2} & \mathrm{t}_{\mathrm{i} 3} & \mathrm{t}_{\mathrm{i} 4} & \mathrm{t}_{\mathrm{i} 5} & \mathrm{t}_{\mathrm{i} 6} \\ \mathrm{r}_{\mathrm{i} 1} & \mathrm{r}_{\mathrm{i} 2} & \mathrm{r}_{\mathrm{i} 3} & \mathrm{r}_{\mathrm{i} 4} & \mathrm{r}_{\mathrm{i} 5} & \mathrm{r}_{\mathrm{i}}\end{array}\right]$. According to Eq.(11), the dimensions of translation and rotation outputs will be $\xi_{\mathrm{ti}}=\sum_{\mathrm{j}=1}^{6} \mathrm{t}_{\mathrm{ij}}$ and $\xi_{\mathrm{ri}}=\sum_{\mathrm{j}=1}^{6} \mathrm{r}_{\mathrm{ij}} \quad(\mathrm{i}=1,2)$, and the non-zero elements of $\mathrm{M}_{\mathrm{Li}}$ imply the corresponding directions.

For convenience of description, the translation and rotation output of leg $\mathrm{I}_{\mathrm{i}}$ are denoted by $\mathrm{G}_{\mathrm{i}}=\left(\xi_{\mathrm{t}}, \mathrm{e}_{\mathrm{i}}\right)$ and $\mathrm{H}_{\mathrm{i}}=\left(\xi_{\mathrm{r}}\right.$, $\left.s_{i}\right)$, where $e_{i}$ and $s_{i}$ are the directions of translation/rotation output of this leg. According to the above definition, $\mathrm{e}_{\mathrm{i}} / \mathrm{s}_{\mathrm{i}}$ can be divided into four different types:

(1) if $\xi_{\mathrm{ti}}=0 / \xi_{\mathrm{ri}}=0$, then $\mathrm{e}_{\mathrm{i}} / \mathrm{s}_{\mathrm{i}}=\Phi$ (empty set);

(2) if $\xi_{\mathrm{ti}}=1 / \xi_{\mathrm{ri}}=1$, then $\mathrm{e}_{\mathrm{i}} / \mathrm{s}_{\mathrm{i}}$ is a spatial line;

(3) if $\xi_{\mathrm{ti}}=2 / \xi_{\mathrm{ri}}=2$, then $\mathrm{e}_{\mathrm{i}} / \mathrm{s}_{\mathrm{i}}$ is a plane;

(4) if $\xi_{\mathrm{ti}}=3 / \xi_{\mathrm{ri}}=3$, then $\mathrm{e}_{\mathrm{i}} / \mathrm{s}_{\mathrm{i}}$ is denoted as "-" (arbitrary in space). 
Suppose the intersection result is described as $M_{p a(1 \sim 2)}=\left[\begin{array}{lllllll}t_{1} & t_{2} & t_{3} & t_{4} & t_{5} & t_{6} \\ r_{1} & r_{2} & r_{3} & r_{4} & r_{5} & r_{6}\end{array}\right]\left[\begin{array}{l}1 j\end{array}\right](j=1$ or 2), where $j$ is the number of reference leg.

Similarly, the dimension of translation/rotation output is denoted by $\xi_{t(1 \sim 2)}=\sum_{\mathrm{i}=1}^{6} \mathrm{t}_{\mathrm{i}} / \xi_{\mathrm{r}(1 \sim 2)}=\sum_{\mathrm{i}=1}^{6} \mathrm{r}_{\mathrm{i}}$.

The translation and rotation output can be expressed as $G_{(1 \sim 2)}=\left(\xi_{t(1 \sim 2)}, e_{(1 \sim 2)}\right)$ and $H_{(1 \sim 2)}=\left(\xi_{r(1 \sim 2)}, s_{(1 \sim 2)}\right)$, where $e_{(1 \sim 2)}$ and $\mathrm{s}_{\left(1^{\sim} 2\right)}$ are the corresponding directions.

Note that the legs $L_{1}$ and $L_{2}$, which were used to introduce the computation algorithm, can be either the legs constituting a PM or a sub-PM composed by several legs. Similarly, the result of the above intersection operation can also be regarded as the POC of a sub-PM continuing to participate in other operations. The intersection output of matrices of two POCs can be calculated following these two rules.

\subsubsection{Intersection rules for translation output of POCS}

In the process of intersection operation, since the rotation outputs have no influence on the translation outputs, the translation outputs can be intersected seperately. Actually, the intersection operation of translations of two legs is to solve $G_{(1 \sim 2)}=G_{1} \cap G_{2}$, which can be obtained according the rules shown in Table 5 .

Table 5 Intersection rules for translation output of POCs

\begin{tabular}{|c|c|c|c|c|c|c|}
\hline \multirow{2}{*}{$\mathrm{L}_{2}$} & \multirow{2}{*}{$\xi_{\mathrm{t} 1}=0$} & \multicolumn{2}{|r|}{$\xi_{\mathrm{t} 1}=1$} & \multicolumn{2}{|r|}{$\xi_{\mathrm{t} 1}=2$} & \multirow{2}{*}{$\xi_{t 1}=3$} \\
\hline & & $e_{1}|| e_{2}$ & $\mathrm{e}_{1} \mathrm{He}_{2}$ & $e_{1} \| e_{2}$ & $\mathrm{e}_{1} \mathrm{He}_{2}$ & \\
\hline$\xi_{\mathrm{t} 2}=0$ & $\mathrm{G}_{2}$ & & $\mathrm{G}_{2}$ & & $\mathrm{G}_{2}$ & $\mathrm{G}_{2}$ \\
\hline$\xi_{\mathrm{tz}}=1$ & $\mathrm{G}_{1}$ & $\mathrm{G}_{2}$ & $\xi_{t(1 \sim 2)}=0, e_{(1 \sim 2)}=\Phi$ & $\mathrm{G}_{2}$ & $\xi_{t(1 \sim 2)}=0, e_{(1 \sim 2)}=\Phi$ & $\mathrm{G}_{2}$ \\
\hline$\xi_{\mathrm{t} 2}=2$ & $\mathrm{G}_{1}$ & $\mathrm{G}_{1}$ & $\xi_{t(1 \sim 2)}=0, e_{(1 \sim 2)}=\Phi$ & $\mathrm{G}_{2}$ & $\xi_{t(1 \sim 2)}=1, e_{(1 \sim 2)}=e_{1} \cap e_{2}$ & $\mathrm{G}_{2}$ \\
\hline$\xi_{\mathrm{t} 2}=3$ & $\mathrm{G}_{1}$ & & $\mathrm{G}_{1}$ & & $\mathrm{G}_{1}$ & $\mathrm{G}_{1}$ \\
\hline
\end{tabular}

\subsubsection{Intersection rules for rotation output of POCs}

Similarly, the intersection of rotational output of two POC matrices is to solve $\mathrm{H}_{(1 \sim 2)}=\mathrm{H}_{1} \cap \mathrm{H}_{2}$. However, as stated above, translation outputs affect the position of the rotation axis. Therefore, the translaotional outputs of a leg must be taken into account before establishing the intersection rules of the rotational output of POCs.

It is well known, if a leg has 2-dimensional translations and 1-dimensional rotation perpendicular to the 2D translation plane, no matter where the revolute joint is, the end link of the leg can rotate around any axis to be perpendicular to the $2 \mathrm{D}$ plane. Likewise, if a leg has 3-dimensional translations and rotations, no matter where the rotation joints are, the end link of the leg can rotate around any axes parallel to the rotational joints. This type of rotational output is called as a fully-free output (Denoted as FF-type), and anything else is called a non-fully-free output (Denoted as non-FF-type). Generally, for the 3-DOF non-FF-type rotation type, it is usually caused by pair S, so it is considered that the three axes intersect at one point, i.e., the spherical center.

On the basis of defining the type of rotational output, the intersection operation rules for rotation output can be divided into three types and listed in Table 6 . It is noted that, the symbol "*" in the table indicates that the three axes have intersection points, and the superscript "a" indicates that the output axis will pass through the intersection point.

It should be noted that the number of the reference leg $\left(\mathrm{l}_{\mathrm{j}}\right)$ can be concluded directly from the result of the intersection operations. As it can be seen from Table 5 and Table 6 , the result of $G_{1}$ or $G_{2}$ indicates that $l_{j}$ is $L_{1}$ or $L_{2}$ correspondingly. In particular, when the result of intersection is not capable of motion, the output will be shown as' $\Phi^{\prime}$ (Empty set). 
Table 6 Intersection rules for rotation output

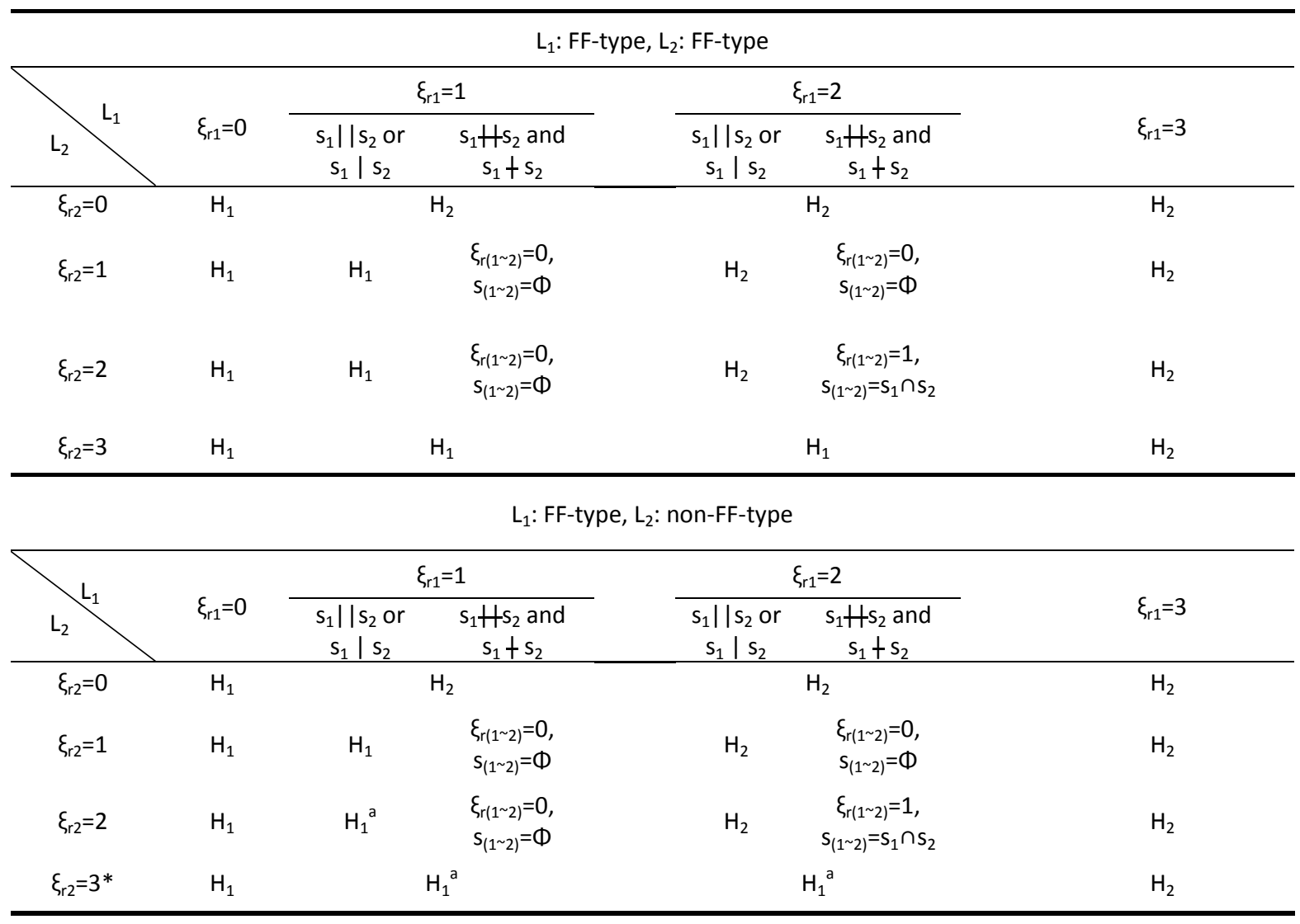

$\mathrm{L}_{1}$ : non-FF-type, $\mathrm{L}_{2}$ : non-FF-type

\begin{tabular}{|c|c|c|c|c|c|c|c|}
\hline \multirow{2}{*}{$\mathrm{L}_{2}^{\mathrm{L}_{1}}$} & \multirow{2}{*}{$\xi_{\mathrm{r} 1}=0$} & \multicolumn{2}{|c|}{$\xi_{\mathrm{r} 1}=1$} & \multicolumn{2}{|c|}{$\xi_{\mathrm{r} 1}=2$} & \multicolumn{2}{|c|}{$\xi_{\mathrm{r} 1}=3^{*}$} \\
\hline & & $\mathrm{s}_{1} \mid \mathrm{s}_{2}$ & $s_{1}+s_{2}$ & $\mathrm{~s}_{1} \mid \mathrm{s}_{2}$ & $s_{1}+s_{2}$ & $s_{1} \mid s_{2}$ & $s_{1}+s_{2}$ \\
\hline$\xi_{r 2}=0$ & $\mathrm{H}_{1}$ & \multicolumn{2}{|c|}{$\mathrm{H}_{2}$} & \multicolumn{2}{|c|}{$\mathrm{H}_{2}$} & \multicolumn{2}{|c|}{$\mathrm{H}_{2}$} \\
\hline$\xi_{\mathrm{r} 2}=1$ & $\mathrm{H}_{1}$ & $\mathrm{H}_{1}$ & $\begin{array}{l}\xi_{r(1 \sim 2)}=0 \\
s_{(1 \sim 2)}=\Phi\end{array}$ & $\mathrm{H}_{2}$ & $\begin{array}{l}\xi_{r(1 \sim 2)}=0 \\
s_{(1 \sim 2)}=\Phi\end{array}$ & $\mathrm{H}_{2}$ & $\begin{array}{l}\xi_{r(1 \sim 2)}=0 \\
S_{(1 \sim 2)}=\Phi\end{array}$ \\
\hline$\xi_{\mathrm{r} 2}=2$ & $\mathrm{H}_{1}$ & $\mathrm{H}_{1}$ & $\begin{array}{l}\xi_{r(1 \sim 2)}=0 \\
s_{(1 \sim 2)}=\Phi\end{array}$ & $\mathrm{H}_{2}$ & $\begin{array}{l}\xi_{r(1 \sim 2)}=0 \\
s_{(1 \sim 2)}=\Phi\end{array}$ & $\mathrm{H}_{2}{ }^{\mathrm{a}}$ & $\begin{array}{l}\xi_{r(1 \sim 2)}=0 \\
S_{(1 \sim 2)}=\Phi\end{array}$ \\
\hline$\xi_{\mathrm{r} 2}=3^{*}$ & $\mathrm{H}_{1}$ & $\mathrm{H}_{1}{ }^{\mathrm{a}}$ & $\begin{array}{l}\xi_{r(1 \sim 2)}=0 \\
S_{(1 \sim 2)}=\Phi\end{array}$ & $\mathrm{H}_{1}$ & $\begin{array}{l}\xi_{\mathrm{r}(1 \sim 2)}=0 \\
\mathrm{~s}_{(1 \sim 2)}=\Phi\end{array}$ & $\mathrm{H}_{2}$ & $\begin{array}{c}\xi_{\mathrm{r}(1 \sim 2)}=1, \\
\mathrm{~s}_{(1 \sim 2)}=\mathrm{O}_{1} \mathrm{O}_{2}\end{array}$ \\
\hline
\end{tabular}

\subsubsection{Analysis algorithm procedure for the POC of a PM}

In accordance with the analysis above, the fully-automatic POC analysis procedure of PMs is as follows.

Step 1 Input the number of legs $n$ and POC matrix of each leg $M_{L i}(i=1 \sim n)$ including two virtual legs of the two platforms $\mathrm{M}_{\mathrm{MP}}$ and $\mathrm{M}_{\mathrm{LP}}$.

Step 2 Choose the $1^{\text {st }}$ and the $2^{\text {nd }}$ legs to form a sub-PM denoted as sub-PM $M_{(1 \sim 2)}$.

Step 3 Calculate the POC matrix $\mathrm{M}_{\mathrm{pa}(1 \sim 2)}$ of sub-PM $\mathrm{M}_{(1 \sim 2)}$.

- Obtain intersection of translation outputs $G_{(1 \sim 2)}$

- Obtain intersection of rotation outputs $\mathrm{H}_{(1 \sim 2)}$

- Obtain the result of POC $\mathrm{M}_{\mathrm{pa}\left(1^{\sim} 2\right)}$ of the sub-PM$\left(\mathrm{M}_{(1 \sim 2)}\right.$ 
Step 4 Repeat Step 2 and Step 3 for constructing sub-PM( $\mathrm{M}_{(1 \sim 3)}$ (consisted by sub-PM $\mathrm{P}_{(1 \sim 2)}$ and the $3^{\text {rd }}$ leg), ..., sub$\mathrm{PM}_{(1 \sim n)}$ (consisted by sub-PM $\mathrm{P}_{(1 \sim(n-1))}$ and the $\mathrm{n}^{\text {th }}$ leg), and calculation of the corresponding $\mathrm{M}_{\mathrm{pa}(1 \sim 3)}, \ldots, \mathrm{M}_{\mathrm{pa}(1 \sim n)}$.

Step 5 Output the POC result of PM: $M_{p a}=M_{p a(1 \sim n)}$.

Taking the 3-RRC PM for example, the structural sketch is shown in Fig.6(b). The topology matrices of legs, and joints on the two platforms have been described in Section 3.2.1. The POC of 3-RRC PM can be analyzed as follows.

Step 1 Input the number of legs, and POC of each leg.

Obviously the number of legs is $n=3$.

According to Eq.(15), the topology matrices of 3-RRC legs can be determined as

$$
\begin{aligned}
& M_{L i}=\left[\begin{array}{llllll}
2 & 0 & 0 & 0 & 0 & 0 \\
1 & 0 & 0 & 0 & 0 & 0
\end{array}\right] \|\left[\begin{array}{llllll}
0 & 0 & 0 & 1 & 0 & 0 \\
0 & 0 & 0 & 0 & 0 & 0
\end{array}\right] \\
& =\left[\begin{array}{llllll}
2 \mathrm{P} 0 & 0 \mathrm{P} 0 & 0 \mathrm{P} 0 & 0 \mathrm{P} 1 & 0 \mathrm{P} 0 & 0 \mathrm{P} 0 \\
1 \mathrm{P} 0 & 0 \mathrm{P} 0 & 0 \mathrm{P} 0 & 0 \mathrm{P} 0 & 0 \mathrm{P} 0 & 0 \mathrm{P} 0
\end{array}\right]=\left[\begin{array}{llllll}
2 & 0 & 0 & 1 & 0 & 0 \\
1 & 0 & 0 & 0 & 0 & 0
\end{array}\right] \quad(\mathrm{i}=1,2,3) \text {. } \\
& =\left[\begin{array}{llllll}
3 & 0 & 0 & 0 & 0 & 0 \\
1 & 0 & 0 & 0 & 0 & 0
\end{array}\right]=\left[\begin{array}{l}
\mathrm{G}_{\mathrm{i}} \\
\mathrm{H}_{\mathrm{i}}
\end{array}\right]
\end{aligned}
$$

Thus, $\xi_{\mathrm{ti}}=3$ means that there are arbitrary translations in space, and $\xi_{\mathrm{ri}}=1$ is a one-dimension rotation around the $R_{i 1}$ axis in the $i^{\text {th }}$ leg, i.e. $s_{i}=R_{i 1}$.

Step 2 Calculate the POC matrix $\mathrm{M}_{\mathrm{pa}(1 \sim 2)}$ of sub-PM $\mathrm{PM}_{(1 \sim 2)}$ constituted by the $1^{\text {st }}$ and the $2^{\text {nd }}$ legs.

According to Table $5, \xi_{\mathrm{t} 1}=\xi_{\mathrm{t} 2}=3$, then $\xi_{\mathrm{t}(1 \sim 2)}=3$, and $\mathrm{G}_{(1 \sim 2)}=\mathrm{G}_{2}$.

Since $\xi_{\mathrm{r} 1}=\xi_{\mathrm{r} 2}=1, s_{1}=R_{11}, s_{2}=R_{21}$, and $R_{11} H R_{21}$, we obtain $s_{1} H s_{2}$. According to Table 6 , then $\xi_{r(1 \sim 2)}=0$, and $s_{(1 \sim 2)}=\Phi$.

Then, the POC matrix of sub-PM(1 2) is $M_{\text {pa(1 2) }}=\left[\begin{array}{llllll}2 & 0 & 0 & 1 & 0 & 0 \\ 0 & 0 & 0 & 0 & 0 & 0\end{array}\right]=\left[\begin{array}{l}G_{(1 \sim 2)} \\ H_{(1 \sim 2)}\end{array}\right]$.

Step 3 Calculate the POC matrix $\mathrm{M}_{\mathrm{pa}\left(1^{\sim} 3\right)}$ of sub-PM(1 $\mathrm{M}_{\left(1_{3}\right)}$ constituted by the sub-PM $\mathrm{M}_{(1 \sim 2)}$ and the third RRC leg.

According to Table 5, $\xi_{t(1 \sim 2)}=\xi_{\mathrm{t} 3}=3$, then $\xi_{\mathrm{t}(1 \sim 3)}=3$, and $\mathrm{G}_{(1 \sim 3)}=\mathrm{G}_{3}$.

According to Table 6, $\xi_{r(1 \sim 2)}=0, \xi_{r 3}=1$, and $s_{(1 \sim 2)}=\Phi, s_{3}=R_{31}$, then, $\xi_{r(1 \sim 3)}=0, s_{(1 \sim 3)}=\Phi$.

Thus, the POC matrix of sub-PM $(1 \sim 3)$ is $M_{p a(1 \sim 3)}=\left[\begin{array}{llllll}2 & 0 & 0 & 1 & 0 & 0 \\ 0 & 0 & 0 & 0 & 0 & 0\end{array}\right]=\left[\begin{array}{llllll}3 & 0 & 0 & 0 & 0 & 0 \\ 0 & 0 & 0 & 0 & 0 & 0\end{array}\right]=\left[\begin{array}{l}\mathrm{G}_{(1 \sim 3)} \\ \mathrm{H}_{(1 \sim 3)}\end{array}\right]=\left[\begin{array}{c}\mathrm{G}_{3} \\ \Phi\end{array}\right]$

Step 4 Output the result of the POC of this PM

$$
\mathrm{M}_{\mathrm{pa}}=\mathrm{M}_{\mathrm{pa}(1 \sim 3)}=\left[\begin{array}{c}
\mathrm{G}_{3} \\
\Phi
\end{array}\right]
$$

The POC of the 3-RRC PM is a 3-dimensional translation, and this result is consistent with the result in [18].

\subsection{Calculating the number of independent displacement equations}

Eq.(6) shows that solving the number of independent displacement equations of an independent loop involves the union operation of POC matrices. For the legs $L_{1}$ and $L_{2}$ mentioned above, suppose the resulting matrix of the union operation to be

$$
M_{L(1 U 2)}=\left[\begin{array}{llllll}
t_{1} & t_{2} & t_{3} & t_{4} & t_{5} & t_{6} \\
r_{1} & r_{2} & r_{3} & r_{4} & r_{5} & r_{6}
\end{array}\right]
$$

The dimension of independent output is

$$
\xi_{\mathrm{Lt}(1 \mathrm{U} 2)}=\sum_{\mathrm{i}=1}^{6} \mathrm{t}_{\mathrm{i}} / \xi_{\mathrm{Lr}(1 \mathrm{U} 2)}=\sum_{\mathrm{i}=1}^{6} \mathrm{r}_{\mathrm{i}}
$$

The translation output is $\mathrm{G}_{(1 \cup 2)}=\left(\xi_{\mathrm{tt}(1 \cup 2)}, \mathrm{e}_{(1 \cup 2)}\right)$ and the rotation output is $\mathrm{H}_{(1 \cup 2)}=\left(\xi_{\mathrm{Lr}\left(1 U_{2}\right)}, \mathrm{s}_{\left(1 \cup_{2}\right)}\right)$, where $\mathrm{e}_{(1 \cup 2)} / \mathrm{s}_{(1 \cup 2)}$ is the direction of translation/rotation. Thus, the number of independent displacement equations of the independent loop is $\xi_{\mathrm{L}(1 \cup 2)}=\xi_{\mathrm{Lt}(1 \cup 2)}+\xi_{\mathrm{Lr}(1 \cup 2)}$. 


\subsubsection{Rules for the dimension of translation output of SLC}

Solving the dimension of translation output of an SLC means essentially to calculate $\xi_{\mathrm{Lt}(1 \mathrm{U} 2)}=\operatorname{dim}\left(\mathrm{G}_{1} \cup \mathrm{G}_{2}\right)$. The corresponding operation rules are shown in Table 7.

Table 7. Rules for the dimension of the translation output of SLC

\begin{tabular}{|c|c|c|c|c|c|c|}
\hline \multirow{2}{*}{$\mathrm{L}_{2} \mathrm{~L}_{1}$} & \multirow{2}{*}{$\xi_{\mathrm{t} 1}=0$} & \multicolumn{2}{|c|}{$\xi_{t 1}=1$} & \multicolumn{2}{|c|}{$\xi_{t 1}=2$} & \multirow{2}{*}{$\xi_{\mathrm{t} 1}=3$} \\
\hline & & $\mathrm{e}_{1} \| \mathrm{e}_{2}$ & $\mathrm{e}_{1} \mathrm{He}_{2}$ & $\mathrm{e}_{1} \| \mathrm{e}_{2}$ & $\mathrm{e}_{1} \mathrm{He}_{2}$ & \\
\hline$\xi_{\mathrm{t} 2}=0$ & 0 & & & & & 3 \\
\hline$\xi_{\mathrm{t} 2}=1$ & 1 & 1 & 2 & 2 & 3 & 3 \\
\hline$\xi_{\mathrm{t} 2}=2$ & 2 & 2 & 3 & 2 & 3 & 3 \\
\hline$\xi_{\mathrm{t} 2}=3$ & 3 & & & & & 3 \\
\hline
\end{tabular}

\subsubsection{Rules for the dimension of rotation output of SLC}

Similarly, the dimension of the rotation output of SLC is to calculate $\xi_{\operatorname{Lr}(1 \mathrm{UL}}=\operatorname{dim}\left(\mathrm{H}_{1} \cup \mathrm{H}_{2}\right)$, and its operation rules are shown in Table 8.

Table 8. Rules for the dimension of the rotation output of SLC

\begin{tabular}{|c|c|c|c|c|c|c|}
\hline \multirow{2}{*}{$\mathrm{L}_{2}$} & \multirow{2}{*}{$\xi_{r 1}=0$} & \multicolumn{2}{|c|}{$\xi_{r 1}=1$} & \multicolumn{2}{|c|}{$\xi_{r 1}=2$} & \multirow{2}{*}{$\xi_{r 1}=3$} \\
\hline & & $e_{1} \| e_{2}$ & $\mathrm{e}_{1} \mathrm{He}_{2}$ & $e_{1} \| e_{2}$ & $\mathrm{e}_{1} \mathrm{He}_{2}$ & \\
\hline$\xi_{\mathrm{r} 2}=0$ & 0 & & & & & 3 \\
\hline$\xi_{\mathrm{r} 2}=1$ & 1 & 1 & 2 & 2 & 3 & 3 \\
\hline$\xi_{\mathrm{r} 2}=2$ & 2 & 2 & 3 & 2 & 3 & 3 \\
\hline$\xi_{\mathrm{r} 2}=3$ & 3 & & & & & 3 \\
\hline
\end{tabular}

The rules described above does not only work for serial legs but also for equivalent sub-PMs. Therefore, it is emphasized once more that the legs $L_{1}$ and $L_{2}$, which were used to introduce the computation algorithm, can be either the legs constituting a PM or a sub-PM composed by several legs.

\section{Procedure of automatic mobility analysis of PMs}

Based on the POC algorithm for a leg, for a PM and for the number of independent displacement equations, the steps of mobility analysis of PMs are illustrated below.

Step 1 Input topological structure of a PM.

Number $1 \sim(v+1)$ of legs in sequence and input the corresponding topology matrix of each leg as $L_{1}, \ldots, L_{(v+1)}$. Then, input the topology matrix between legs, $L_{M P}$ characterizes a virtual leg constituted by pairs of joints on the moving platform and $L_{B P}$ is analogously defined on the fixed platform. Based on above, the number of joints $f_{i}$ and the number of legs $v+1$ can be obtained automatically.

It should be pointed out that for the PM to be analyzed, the legs can be numbered arbitrarily and does not affect the result of the mobility analysis. However, once the legs are numbered, the order of the two virtual legs should be basic on the number.

Step 2 Select base point $O^{\prime}$ on the moving platform.

Step 3 Calculate POC matrices of $(v+1)$ legs, denoted as $M_{L 1}, \ldots M_{L(v+1)}$.

Step 4 Calculate the sum of all joints: $f_{(1 \sim(v+1))}=\sum_{i=1}^{v+1} f_{i}$. 
Step 5 Calculate the $\mathrm{POC}$ matrix $\mathrm{M}_{\mathrm{pa}\left(1^{\sim j}\right)}$ of the sub-PM$\left(\mathrm{M}_{\left(1^{\sim j}\right)}\right.$ composed by the front $\mathrm{j}^{\text {th }}$ legs connected in parallel $(j=2, \ldots, v)$.

(1) Calculate the translation output $G_{(1 \sim j)}=G_{(1 \sim(j-1))} \cap G_{(j+1)}$.

(2) Calculate the rotation output $\left.\mathrm{H}_{\left(1^{\sim j}\right)}\right)=\mathrm{H}_{(1 \sim(j-1))} \cap \mathrm{H}_{(j+1)}$.

(3) Get $\quad \mathrm{M}_{\mathrm{pa}(1 \sim \mathrm{j})}=\left[\begin{array}{l}\mathrm{G}_{(1 \sim \mathrm{j})} \\ \mathrm{H}_{(1 \sim \mathrm{j})}\end{array}\right]$.

Step 6 Calculate the number of the independent displacement equations $\xi_{j}$ of the $j^{\text {th }}$ loop $S L C_{j}$, which is composed by the sub-PM $\mathrm{P}_{\left(1^{\sim j}\right)}$ and the $(j+1)^{\text {th }}$ leg connected in series $(j=1, \ldots, v)$.

(1) Calculate the dimension of the independent translation output of $S L C_{1}: \xi_{L j \mathrm{t}}=\operatorname{dim}\left(\mathrm{G}_{\left(1^{\sim j j}\right)} \mathrm{UG}_{(j+1)}\right)$.

(2) Calculate the dimension of the independent rotation output of $S L C_{1}: \xi_{L j \mathrm{r}}=\operatorname{dim}\left(\mathrm{H}_{\left(1^{\sim j}\right)} \cup \mathrm{H}_{(j+1)}\right)$.

(3) Calculate $\xi_{\mathrm{Lj}}=\xi_{\mathrm{Lj} \mathrm{t}}+\xi_{\mathrm{Lj} \mathrm{j}}$.

Step 6 Calculate the number of DOF: $\mathrm{F}=\mathrm{f}_{(1 \sim(v+1))}-\sum_{\mathrm{i}=1}^{v} \xi_{\mathrm{i}}$.

Step 7 Determine the mobility property of this $P M: M_{p a}=M_{p a(1 \sim v)}=M_{p a(1 \sim(v-1))} \cap M_{L(v+1)}$.

The corresponding flow process chart of an automatic mobility analysis is shown in Fig.7.

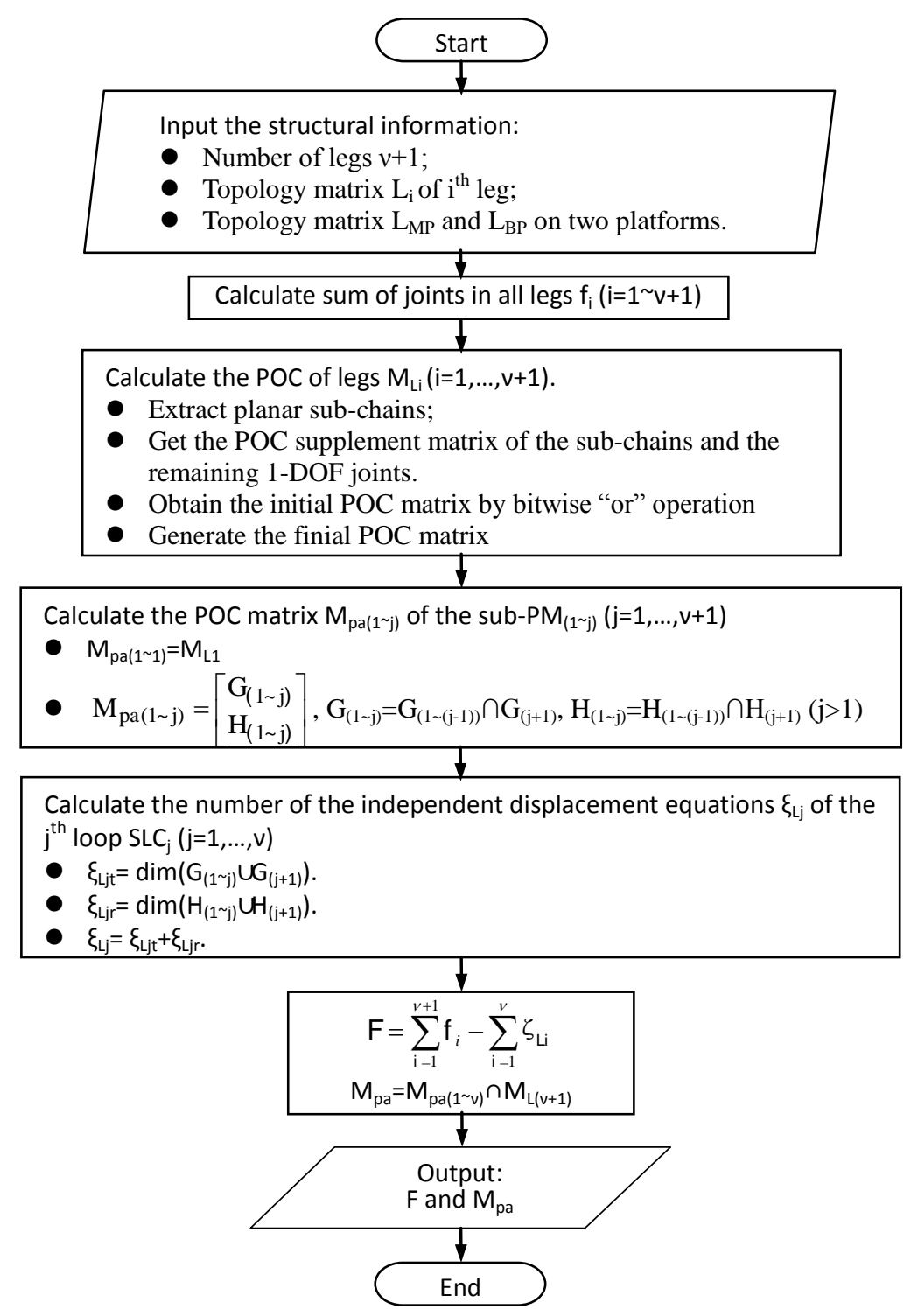


Fig. 7. Procedure of mobility analysis of a PM

\section{Software implementation and case study}

\subsection{Software implementation}

Based on the basic principles, algorithmic rules and algorithmic procedures, a program which can analyze the mobility of PMs automatically has been developed on Windows platform with Visual C++6.0 software and objectoriented programming language. Using the software, a large number of PMs listed in [18] have been analyzed automatically and the effectiveness of the presented method has been testified.

Fig.8 presents the developed human-computer interactive interface. The input information is located at the top of the interactive interface. It mainly includes the number of legs, topological structure matrices of two virtual legs on the two platforms and the topology structure matrices of all legs. It is using integer arrays and is easy to operate.

What needs to be added is that, as the topology matrices are symmetric, only upper triangular elements need to be input in the human-computer interaction interface for convenience.

In addition to DOF and POC (i.e., Number of POC Elements, T-directions and R-directions), other topological features can be obtained, such as independent loops, independent displacement equations. Due to limited space, this work does not go into all details.

\subsection{Case study}

Detailed examples of the mobility analysis of four typical PMs are presented in this section.

\subsubsection{Mobility analysis of Tricept PM}

The Tricept non-overstrained PM shown in Fig.6(a) has 3-DOF which comprise one translation and two rotations. The legs are 3-UPS and 1-UP. The axes of the joints on the two platforms are arbitrary to each other.

(1) Input topological structure matrices of this PM

$\begin{aligned} \text { Topology matrix of UPS leg is } \mathrm{L}_{\mathrm{i}} & =\left[\begin{array}{llllll}8 & 2 & 2 & 0 & 0 & 0 \\ 2 & 8 & 2 & 1 & 0 & 0 \\ 2 & 2 & 9 & 2 & 0 & 0 \\ 0 & 1 & 2 & 8 & 0 & 0 \\ 0 & 0 & 0 & 0 & 8 & 4 \\ 0 & 0 & 0 & 0 & 4 & 8\end{array}\right],(\mathrm{i}=1 \sim 3) \text {. } \\ \text { Topology matrix of UP leg is } \mathrm{L}_{4} & =\left[\begin{array}{lll}8 & 2 & 2 \\ 2 & 8 & 2 \\ 2 & 2 & 9\end{array}\right] \text {. }\end{aligned}$

Topology matrices of two virtual legs on the two platforms $\mathrm{L}_{\mathrm{MP}}$ and $\mathrm{L}_{\mathrm{BP}}$ are respectively, $\mathrm{L}_{\mathrm{MP}}=\left[\begin{array}{llll}8 & 0 & 0 & 0 \\ 0 & 8 & 0 & 0 \\ 0 & 0 & 8 & 0 \\ 0 & 0 & 0 & 9\end{array}\right]$, $\mathrm{L}_{\mathrm{BP}}=\left[\begin{array}{llll}8 & 0 & 0 & 0 \\ 0 & 8 & 0 & 0 \\ 0 & 0 & 8 & 0 \\ 0 & 0 & 0 & 8\end{array}\right]$

Number of legs $v+1=4$.

(2) Select an arbitrary point $\mathrm{O}^{\prime}$ on the moving platform as the base point.

(3) Calculate POC matrices of legs $M_{L 1}, \ldots M_{L 4}$.

(1) $\mathrm{M}_{\mathrm{Li}}=\left[\begin{array}{llllll}3 & 0 & 0 & 0 & 0 & 0 \\ 3 & 0 & 0 & 0 & 0 & 0\end{array}\right]=\left[\begin{array}{l}\mathrm{G}_{\mathrm{i}} \\ \mathrm{H}_{\mathrm{i}}\end{array}\right], \xi_{\mathrm{ri}}=3$, and $\xi_{\mathrm{ti}}=3(\mathrm{i}=1 \sim 3)$

(2) $\mathrm{M}_{\mathrm{L} 4}=\left[\begin{array}{llllll}0 & 0 & 1 & 0 & 0 & 0 \\ 1 & 1 & 0 & 0 & 0 & 0\end{array}\right]=\left[\begin{array}{l}\mathrm{G}_{4} \\ \mathrm{H}_{4}\end{array}\right]$, and $\xi_{\mathrm{t} 4}=1, \xi_{\mathrm{r} 4}=2, \mathrm{e}_{4}=\mathrm{P}_{43}, \mathrm{~s}_{4}=\left(\mathrm{R}_{41}, \mathrm{R}_{42}\right)$.

(4) Get the sum of all joints in this PM: $f_{(1-4)}=6+6+6+3=21$.

(5) Calculate the POC matrices $M_{\text {pa(1 j) }}$ of sub-PM $M_{(1 \sim j)}$ constituted by the front $j$ legs in parallel $(j=1 \sim 3)$.

- Set $\mathrm{M}_{\mathrm{pa}(1 \sim 1)}=\mathrm{M}_{\mathrm{L} 1}$ 
- Calculate the POC matrix $\mathrm{M}_{\mathrm{pa}(1 \sim 2)}$ of sub-PM$\left(\mathrm{M}_{\left(1_{2}\right)}\right.$ constituted by the $1^{\text {st }}$ and the $2^{\text {nd }}$ leg in parallel.

(1) $\xi_{\mathrm{ti}}=3$, as shown in Table $5, \mathrm{G}_{(1 \sim 2)}=\mathrm{G}_{(1 \sim 1)} \cap \mathrm{G}_{2}=\mathrm{G}_{2}(\mathrm{i}=1,2)$.

(2) $\xi_{\mathrm{ri}}=3$, as shown in Table 6, $\mathrm{H}_{(1 \sim 2)}=\mathrm{H}_{(1 \sim 1)} \cap \mathrm{H}_{2}=\mathrm{H}_{2}(\mathrm{i}=1,2)$.

(3) So, $M_{\mathrm{pa}(1 \sim 2)}=\left[\begin{array}{llllll}3 & 0 & 0 & 0 & 0 & 0 \\ 3 & 0 & 0 & 0 & 0 & 0\end{array}\right]=\left[\begin{array}{l}\mathrm{G}_{2} \\ \mathrm{H}_{2}\end{array}\right]$.

- Calculate the POC matrix of sub-PM( $M_{(1 \sim 2)}$ composed by sub-PM $(1 \sim 2)$ and the $3^{\text {rd }}$ leg:

$M_{p a(1 \sim 3)}=M_{p a(1 \sim 2)} \cap M_{L 3}=\left[\begin{array}{l}G_{2} \\ H_{2}\end{array}\right] \cap\left[\begin{array}{l}G_{3} \\ H_{3}\end{array}\right]=\left[\begin{array}{l}G_{3} \\ H_{3}\end{array}\right]$.

(5) Calculate the number of the independent displacement equations $\xi_{\mathrm{Lj}}$ of $\mathrm{SLC}_{\mathrm{j}}(\mathrm{j}=1 \sim 3)$.

- Calculate $\xi_{\mathrm{L} 1}$ of the first loop $\mathrm{SLC}_{1}$ concatenated by $1^{\text {st }}$ and $2^{\text {nd }} \operatorname{leg}, \xi_{\mathrm{L} 1}=\operatorname{dim}\left(\mathrm{M}_{\mathrm{pa}(1 \sim 1)} \cup \mathrm{M}_{\mathrm{L} 2}\right)$.

(1) $\xi_{\mathrm{ti}}=3$, as shown in Table $7, \xi_{\mathrm{L} 1 \mathrm{t}}=3(\mathrm{i}=1,2)$.

(2) $\xi_{\mathrm{ri}}=3$, as shown in Table $8, \xi_{\mathrm{L} 1 \mathrm{r}}=3(\mathrm{i}=1,2)$.

(3) So, $\xi_{L 1}=\xi_{L 1 t}+\xi_{L 1 \mathrm{r}}=3+3=6$.

- Calculate $\xi_{\mathrm{L} 2}$ of $\mathrm{SLC}_{2}: \xi_{\mathrm{L} 2}=\operatorname{dim}\left(\mathrm{M}_{\mathrm{pa}(1 \sim 2)} \mathrm{UM}_{\mathrm{L} 3}\right)$.

(1) $\xi_{\mathrm{t}(1 \cap 2)}=3$, and $\xi_{\mathrm{t} 3}=3$, then $\xi_{\mathrm{t} 2 \mathrm{t}}=3$.

(2) $\xi_{\mathrm{r}(1 \cap 2)}=3$, and $\xi_{\mathrm{r} 3}=3$, then $\xi_{\mathrm{L} 2 \mathrm{r}}=3$.

(3) thus, $\xi_{\mathrm{L} 2}=\xi_{\mathrm{L} 2 \mathrm{t}}+\xi_{\mathrm{L} 2 \mathrm{r}}=3+3=6$.

- Similarly, the number of the independent displacement equations of $\mathrm{SLC}_{3}$ is $\xi_{\mathrm{L} 3}=\xi_{\mathrm{L} 3 \mathrm{t}}+\xi_{\mathrm{L} 3 \mathrm{r}}=3+3=6$.

(6) Get the number of DOF: $F=f_{(1 \sim 4)}-\sum_{i=1}^{3} \xi_{i}=21-(6+6+6)=3$.

(7) Get the mobility property:

$$
M_{p a}=M_{p a(1 \sim 3)} \cap M_{L 4}=\left[\begin{array}{l}
G_{3} \\
H_{3}
\end{array}\right] \cap\left[\begin{array}{l}
G_{4} \\
H_{4}
\end{array}\right]=\left[\begin{array}{l}
G_{4} \\
H_{4}
\end{array}\right]
$$

The results show that this $P M$ has $1 T 2 R$ output, the rotation directions are around $R_{41}$ and $R_{42}$, and the translation direction is along the $\mathrm{P}_{43}$ joint. The result generated by the software is shown in Fig.8, which is consistent with the result in [18].

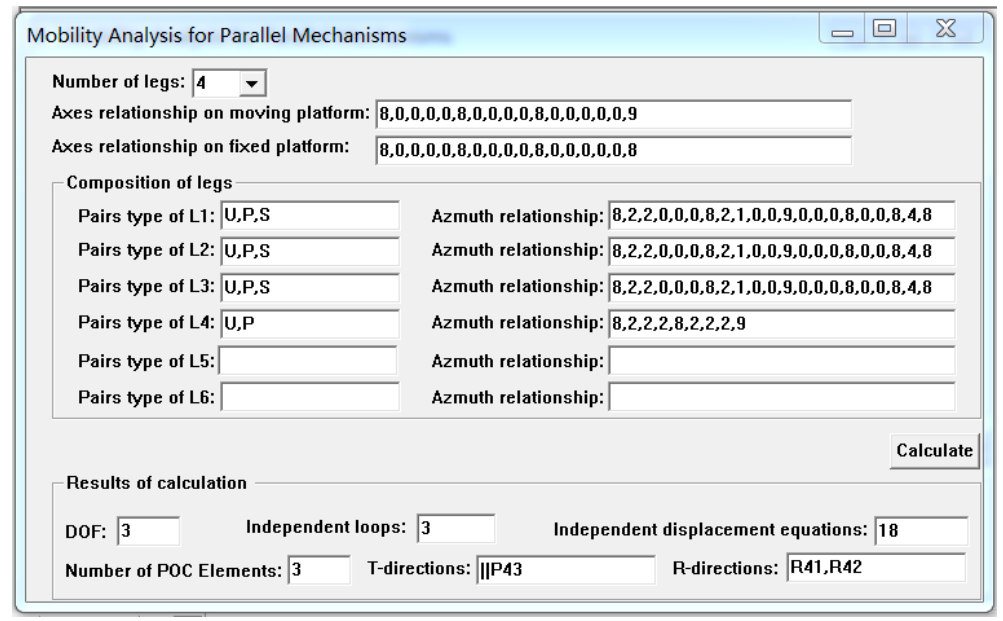

Fig. 8. Mobility analysis of Tricept

\subsubsection{Mobility analysis of 3-RRC PM}

Fig.6(b) shows a 3-RRC PM with overstrains. It has three identical RRC legs connecting the two platforms, labeled with the numbers $1 \sim 3$ respectively. The topological structure of this $P M$ is $P M=\left(L_{1}, L_{2}, L_{3}, L_{M P}, L_{B P}\right)$. The fixed platform is a triangle, and the axes of the joints on the two platforms are all coplanar.

(1) Input topology matrices of the PM 
Topology matrix of RRC leg is $\mathrm{L}_{\mathrm{i}}=\left[\begin{array}{llll}8 & 1 & 1 & 1 \\ 1 & 8 & 1 & 1 \\ 1 & 1 & 8 & 1 \\ 1 & 1 & 1 & 9\end{array}\right],(\mathrm{i}=1 \sim 3)$.

Topology matrices of two virtual legs on the two platforms $\mathrm{L}_{\mathrm{MP}}$ and $\mathrm{L}_{\mathrm{BP}}$ are $\mathrm{L}_{\mathrm{MP}}=\left[\begin{array}{lll}9 & 5 & 5 \\ 5 & 9 & 5 \\ 5 & 5 & 9\end{array}\right]$ and $\mathrm{L}_{\mathrm{BP}}=\left[\begin{array}{lll}8 & 5 & 5 \\ 5 & 8 & 5 \\ 5 & 5 & 8\end{array}\right]$ respectively.

Number of legs $v+1=3$.

(2) Select an arbitrary point $\mathrm{O}^{\prime}$ on the moving platform as the base point.

(3) Calculate POC matrix of legs $M_{L 1}, M_{L 2}$ and $M_{L 3}$.

(1) Recognize and sequentially extract planar and spherical sub-chains: one $G_{3}$ sub-chain $R / / R / / R$ and one 1DOF P joint.

(2) Generate the POC supplemented matrices of $\mathrm{G}_{3}$ and $\mathrm{P}$ as $\mathrm{M}_{\mathrm{G} 3}=\left[\begin{array}{llllll}2 & 0 & 0 & 0 & 0 & 0 \\ 1 & 0 & 0 & 0 & 0 & 0\end{array}\right]$ and $\mathrm{M}_{\mathrm{P}}=\left[\begin{array}{llllll}0 & 0 & 0 & 1 & 0 & 0 \\ 0 & 0 & 0 & 0 & 0 & 0\end{array}\right]$.

(3) Carry out "or" operation on POC matrices in the leg $\mathrm{M}_{\mathrm{Li}}=\left[\begin{array}{llllll}2 & 0 & 0 & 1 & 0 & 0 \\ 1 & 0 & 0 & 0 & 0 & 0\end{array}\right]=\left[\begin{array}{llllll}3 & 0 & 0 & 0 & 0 & 0 \\ 1 & 0 & 0 & 0 & 0 & 0\end{array}\right], \xi_{\mathrm{r}}^{0}=1$, and $\xi_{\mathrm{t}}^{0}=3$ $(i=1 \sim 3)$

(4) $\xi_{\mathrm{t}}=3$ and $\xi_{\mathrm{r}}=1$, then $\mathrm{M}_{\mathrm{Li}}=\left[\begin{array}{llllll}3 & 0 & 0 & 0 & 0 & 0 \\ 1 & 0 & 0 & 0 & 0 & 0\end{array}\right],(\mathrm{i}=1 \sim 3)$. That is to say, $\xi_{\mathrm{ti}}=3$ indicates that the leg has three translation outputs along arbitrary directions, $\xi_{\mathrm{ri}}=1$ indicates that it has one rotation output. $\mathrm{r}_{\mathrm{i} 1}=1$ means that the rotation is around the axes of $R_{i 1}$, i.e. $s_{i}=R_{i 1}$.

(4) Calculate the sum of all joints in this $P M: f_{(1-3)}=4+4+4=12$.

(5) Calculate the POC matrix $M_{p a(1 \sim j)}$ of sub-PM $M_{(1 \sim j)}$ constituted by the front $j$ legs in parallel.

- Set $\mathrm{M}_{\mathrm{pa}(1 \sim 1)}=\mathrm{M}_{\mathrm{L} 1}$

- Calculate the POC matrix $M_{p a(1 \sim 2)}$ of sub-PM $M_{(1 \sim 2)}$ with $M_{p a(1 \sim 2)}=M_{p a(1 \sim 1)} \cap M_{L 2}$.

(1) $\xi_{\mathrm{ti}}=3$, as shown in Table $5, \mathrm{G}_{(1 \sim 2)}=\mathrm{G}_{2}(\mathrm{i}=1,2)$.

(2) $\xi_{\mathrm{ri}}=1$, and $\mathrm{s}_{1} H \mathrm{~s}_{2}$, as shown in Table 6, $\mathrm{H}_{(1 \sim 2)}=\Phi(\mathrm{i}=1,2)$.

(3) So,. $M_{\mathrm{pa}(1 \sim 2)}=\left[\begin{array}{c}\mathrm{G}_{2} \\ \Phi\end{array}\right]$

(6) Calculate the number of the independent displacement equations $\xi_{\mathrm{Lj}}$ of $\mathrm{SLC}_{\mathrm{j}}(\mathrm{j}=1 \sim 2)$.

- Calculate $\xi_{\mathrm{L} 1}$ of the first loop $\mathrm{SLC}_{1}$ concatenated by $1^{\text {st }}$ and $2^{\text {nd }} \mathrm{leg}$.

(1) $\xi_{\mathrm{ti}}=3$, as shown in Table $7, \xi_{\mathrm{L} 1 \mathrm{t}}=3(\mathrm{i}=1,2)$.

(2) $\xi_{\mathrm{ri}}=1$, as shown in Table 8 , and $\mathrm{s}_{1} \mathrm{H} \mathrm{s}_{2}$, then, $\xi_{\mathrm{L} 1 \mathrm{r}}=2(\mathrm{i}=1,2)$.

(3) So, $\xi_{\mathrm{L} 1}=\xi_{\mathrm{L} 1 \mathrm{t}}+\xi_{\mathrm{L} 1 \mathrm{r}}=3+2=5$.

- Calculate the number of the independent displacement equations $\xi_{\mathrm{L} 2}$ of $\operatorname{SLC}_{2}\left(\xi_{\mathrm{L} 2}=\operatorname{dim}\left(\mathrm{M}_{\mathrm{pa}(1 \sim 2)} \mathrm{UM}_{\mathrm{L} 3}\right)\right)$

(1) $\xi_{\mathrm{t} 3}=3$, and $\mathrm{G}_{(1 \sim 2)}=\mathrm{G}_{2}$, as shown in Table 6, $\xi_{\mathrm{L} 2 \mathrm{t}}=3$.

(2) $\xi_{\mathrm{r} 3}=1$, and $\mathrm{H}_{(1 \sim 2)}=\Phi$, as shown in Table $7, \xi_{\mathrm{L} 2 \mathrm{r}}=1$.

(3) Thus, $\xi_{\mathrm{L} 2}=\xi_{\mathrm{L} 2 \mathrm{t}}+\xi_{\mathrm{L} 2 \mathrm{r}}=3+1=4$.

(7) Get the number of DOF: $\mathrm{F}=\mathrm{f}(1 \sim 3)-\sum_{i=1}^{2} \xi_{\mathrm{i}}=12-(5+4)=3$

(8) Get the mobility property: $M_{p a}=M_{p a(1-3)}=M_{p a(1-2)} \cap M_{L 3}=\left[\begin{array}{c}G_{3} \\ \Phi\end{array}\right]$.

It shows that this PM has three translations and the DOF is three also. The automatically generated result is shown in Fig.9. and the result is consistent with the result in [18]. 


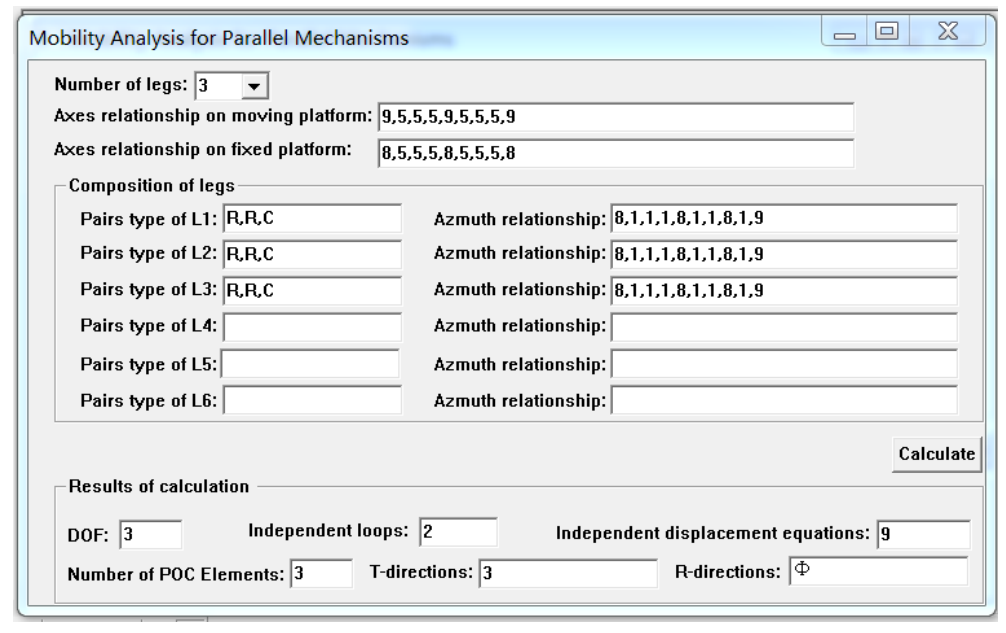

Fig. 9. Mobility analysis of 3-RRC

\subsubsection{Mobility analysis of Exechon}

Fig.6(c) shows an Exechon PM with parasitic motion. It has three identical RPS legs, labeled with the numbers $1 \sim 3$ respectively. The topological structure is $P M=\left(L_{1}, L_{2}, L_{3}, L_{M P}, L_{B P}\right)$. The axes of the joints on the two platforms are all arbitrary.

(1) Input topology matrices of the PM

Topology matrix of RPS leg is $\mathrm{L}_{\mathrm{i}}=\left[\begin{array}{lllll}8 & 2 & 1 & 0 & 0 \\ 2 & 9 & 2 & 0 & 0 \\ 1 & 2 & 8 & 0 & 0 \\ 0 & 0 & 0 & 8 & 4 \\ 0 & 0 & 0 & 4 & 8\end{array}\right]_{(\mathrm{i})} \quad(\mathrm{i}=1 \sim 3)$.

where, the subscript i represents the No. of the intersecting point of the last two revolute joints in the $i^{\text {th }}$ leg.

Topology matrices of two virtual legs on the two platforms are $\mathrm{L}_{\mathrm{MP}}=\left[\begin{array}{lll}8 & 0 & 0 \\ 0 & 8 & 0 \\ 0 & 0 & 8\end{array}\right]$ and $\mathrm{L}_{\mathrm{BP}}=\left[\begin{array}{lll}8 & 0 & 0 \\ 0 & 8 & 0 \\ 0 & 0 & 8\end{array}\right]$, respectively. Number of legs is $3(=v+1)$.

(2) Select center $\mathrm{O}_{13}$ of $\mathrm{S}_{13}$ as the base point $\mathrm{O}^{\prime}$.

(3) Calculate POC matrix of legs $M_{L 1}, M_{L 2}$ and $M_{L 3}$.

(1) Recognize and sequentially extract planar and spherical sub-chains: one $G_{3}$ sub-chain $R \perp P / / R$ and one $S_{2}$ sub-chain through center $\mathrm{O}_{\mathrm{i} 3}(\mathrm{i}=1 \sim 3)$ respectively.

(2) Generate the POC supplemented matrices of $\mathrm{G}_{3}$ and $\mathrm{S}_{2}$ as the follows,

For the first leg: $\mathbf{M}_{\mathrm{G} 3}=\left[\begin{array}{llllll}2 & 0 & 0 & 0 & 0 & 0 \\ 1 & 0 & 0 & 0 & 0 & 0\end{array}\right]$ and $\mathbf{M}_{\mathrm{S} 2}=\left[\begin{array}{llllll}0 & 0 & 0 & 0 & 0 & 0 \\ 0 & 0 & 0 & 1 & 1 & 0\end{array}\right]+\left[\begin{array}{c}0 \\ \# 1\end{array}\right]$.

For the second (and third) leg: $\mathbf{M}_{\mathrm{G} 3}=\left[\begin{array}{llllll}2 & 0 & 0 & 0 & 0 & 0 \\ 1 & 0 & 0 & 0 & 0 & 0\end{array}\right]$ and $\mathrm{M}_{\mathrm{S} 2}=\left[\begin{array}{llllll}0 & 0 & 0 & 0 & 0 & 0 \\ 0 & 0 & 0 & 1 & 1 & 0\end{array}\right]+\left[\begin{array}{c}2 \\ \# \mathrm{i}\end{array}\right](\mathrm{i}=2$ or 3).

Among which, "\#i" indicates the intersecting point of the last two $\mathrm{R}$ joints in the $\mathrm{i}^{\text {th }}$ leg, and the number 2 means two derivative translation outputs in the plane perpendicular to the line connecting point $\mathrm{O}^{\prime}$ and $\mathrm{O}_{\mathrm{i} 3}$.

(3) Carry out "or" operation on POC matrices

To the first leg: $\mathrm{M}_{\mathrm{L} 1}=\left[\begin{array}{cccccc}2 & 0 & 0 & 0 & 0 & 0 \\ 1 & 0 & 0 & 1 & 1 & 0\end{array}\right]_{(1)}, \xi_{\mathrm{r}}^{0}=3, \xi_{\mathrm{t}}^{0}=2$ and $\rho_{\mathrm{i}}=\mathrm{O}^{\prime} \mathrm{O}_{13}$;

To the second and the third legs: $\mathrm{M}_{\mathrm{Li}}=\left[\begin{array}{cccccc}2 & 0 & 0 & 0 & 0 & 0 \\ 1 & 0 & 0 & 1 & 1 & 0\end{array}\right]+\left[\begin{array}{c}1 \\ \# \mathrm{i}\end{array}\right], \xi_{\mathrm{r}}^{0}=3, \xi_{\mathrm{t}}^{0}=3$ and $\rho_{\mathrm{i}}=\mathrm{O}^{\prime} \mathrm{O}_{\mathrm{i} 3}(\mathrm{i}=2,3)$

(4) Then, $\mathrm{M}_{\mathrm{L} 1}=\left[\begin{array}{llllll}2 & 0 & 0 & 0 & 0 & 0 \\ 3 & 0 & 0 & 0 & 0 & 0\end{array}\right]$ and $\mathrm{M}_{\mathrm{Li}}=\left[\begin{array}{llllll}3 & 0 & 0 & 0 & 0 & 0 \\ 3 & 0 & 0 & 0 & 0 & 0\end{array}\right],(\mathrm{i}=2,3)$. i.e., $\xi_{\mathrm{t} 1}=2, \xi_{\mathrm{t} 2}=\xi_{\mathrm{t} 3}=3, \xi_{\mathrm{ri}}=3$ (i=1 3), and $e_{1}=\perp\left(R_{11}\right)$.

(4) Calculate the sum of all joints in this PM: $f_{(1-3)}=5+5+5=15$. 
(5) Calculate the POC matrix $\mathrm{M}_{\mathrm{pa}\left(1^{\sim j}\right)}$ of sub-PM $\mathrm{M}_{\left(1^{\sim j}\right)}$ constituted by the front $\mathrm{j}$ legs in parallel.

- Set $\mathrm{M}_{\mathrm{pa}(1 \sim 1)}=\mathrm{M}_{\mathrm{L} 1}$

- Calculate the $P O C$ matrix $\mathrm{M}_{\mathrm{pa}(1 \sim 2)}$ of sub-PM $\mathrm{M}_{(1 \sim 2)}$ with $\mathrm{M}_{\mathrm{pa}(1 \sim 2)}=\mathrm{M}_{\mathrm{pa}(1 \sim 1)} \cap \mathrm{M}_{\mathrm{L} 2}$.

(1) $\xi_{\mathrm{t} 1}=2$, and $\xi_{\mathrm{t} 2}=3$, as shown in Table $5, \xi_{\mathrm{t}(1 \sim 2)}=2$, and $\mathrm{e}_{(1 \sim 2)}=\mathrm{e}_{1}$.

(2) $\xi_{\mathrm{r} 1}=3, \xi_{\mathrm{r} 2}=3$, as shown in Table $6, \xi_{\mathrm{r}(1 \sim 2)}=3$.

(3) So, $\mathrm{M}_{\mathrm{pa}(1 \sim 2)}=\left[\begin{array}{c}G_{1} \\ H_{1}\end{array}\right]$.

(6) Calculate the number of the independent displacement equations $\xi_{\mathrm{L} j}$ of $\operatorname{SLC}_{\mathrm{j}}(\mathrm{j}=1 \sim 2)$.

- Calculate $\xi_{\mathrm{L} 1}$ of the first loop SLC ${ }_{1}$ concatenated by the $1^{\text {st }}$ and the $2^{\text {nd }}$ leg.

(1) $\xi_{\mathrm{t} 1}=2$, and $\xi_{\mathrm{t} 2}=3$, as shown in Table 7, $\xi_{\mathrm{Lt} 1}=3$.

(2) $\xi_{\mathrm{r} 1}=3, \xi_{\mathrm{r} 2}=3$, as shown in Table 8, then $\xi_{\mathrm{L} 1 \mathrm{r}}=3$.

(3) So, $\xi_{\mathrm{L1}}=\xi_{\mathrm{LIt}}+\xi_{\mathrm{LIr}}=3+3=6$.

- Calculate the number of the independent displacement equations $\xi_{L_{2}}$ of $\operatorname{SLC}_{2}\left(\xi_{L_{2}}=\operatorname{dim}\left(M_{p a(1 \sim 2)} \cup M_{L_{3}}\right)\right)$

(1) $\xi_{\mathrm{t} 3}=3$, and $\mathrm{e}_{(1 \sim 2)}=\mathrm{G}_{1}$, as shown in Table 7, $\xi_{\mathrm{Ltt}}=3$.

(2) $\xi_{\mathrm{r} 3}=3$, and $\xi_{\mathrm{r}(1 \sim 2)}=3$, as shown in Table $8, \xi_{\mathrm{L2r}}=3$.

(3) Thus, $\xi_{\mathrm{L} 2}=\xi_{\mathrm{Ltt}}+\xi_{\mathrm{L} 2 \mathrm{r}}=3+3=6$.

(7) Get the number of DOF: $\quad \mathrm{F}=\mathrm{f}(1 \sim 3)-\sum_{i=1}^{2} \xi_{\mathrm{i}}=15-(6+6)=3$

(8) Get the mobility property: $\mathrm{M}_{\mathrm{pa}}=\mathrm{M}_{\mathrm{pa}(1-2)} \cap \mathrm{M}_{\mathrm{L} 3}=\left[\begin{array}{l}G_{1} \\ H_{1}\end{array}\right] \mathrm{I}\left[\begin{array}{llllll}3 & 0 & 0 & 0 & 0 & 0 \\ 3 & 0 & 0 & 0 & 0 & 0\end{array}\right]=\left[\begin{array}{l}G_{1} \\ H_{1}\end{array}\right]$.

The automatically generated result is shown in Fig.10 and the number of POC elements is 5. Since DOF of this PM is three, the POC has three independent elements and the other two are non-independent. The result is consistent with that in [18], where the axes of rotations outputs are selected as $r_{(013-023)}^{1} u r_{(013-023)}^{1} u r_{(||<\$ 013,023,033))}^{1}$.

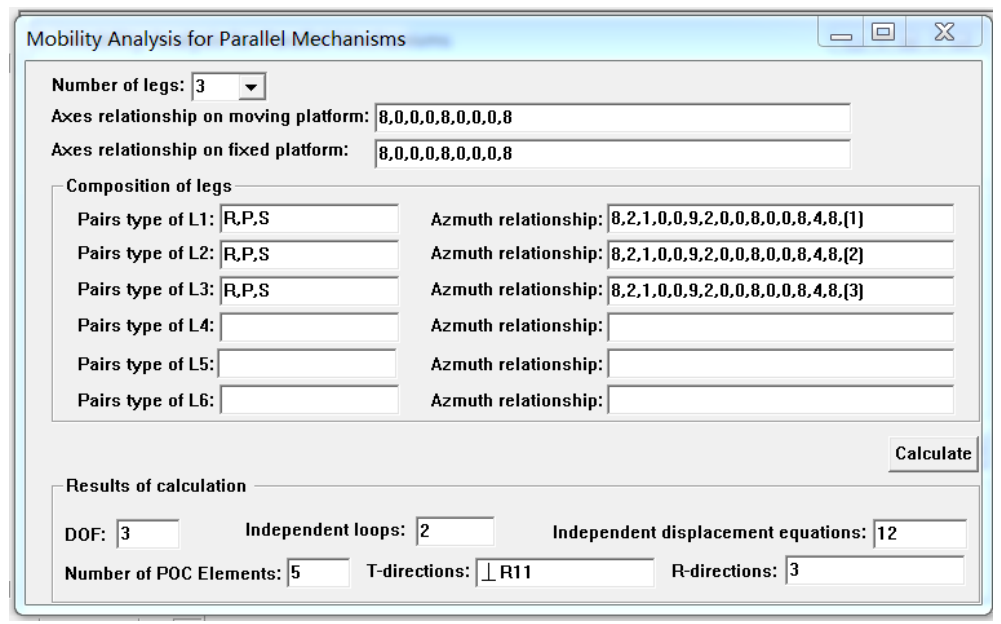

Fig. 10. Mobility analysis of Exechon

\subsubsection{Mobility analysis of 4-RPRRR PM}

Fig.11(a) shows a kind of 4-RPRRR PM. It has four identical $R(\perp P)|| R-R^{*} R$ legs, labeled with the numbers $1 \sim 4$ respectively. The topological structure of this $P M$ is $P M=\left(L_{1}, L_{2}, L_{3}, L_{4}, L_{M P}, L_{B P}\right)$. The axes of the joints on the fixed platforms: $R_{11}|| R_{21}, R_{31}|| R_{41}, R_{11} \perp R_{31}$. Axes of $R_{14}, R_{15}, R_{44}$ and $R_{45}$ intersect at point $O_{1}^{\prime}$, and axes of $R_{24}, R_{25}, R_{34}$ and $\mathrm{R}_{35}$ intersect at point $\mathrm{O}_{2}^{\prime}$. 


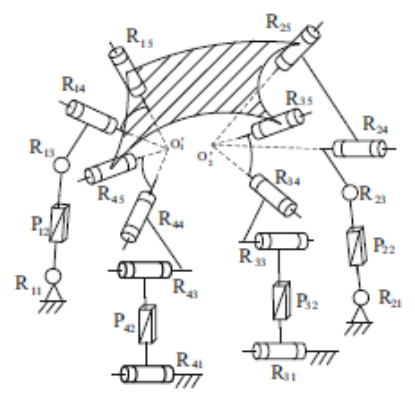

(a)

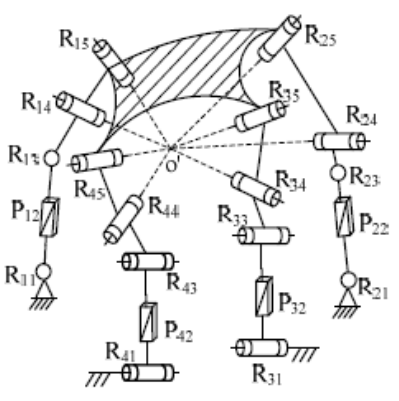

(b)

Fig. 10. Two kinds of 4-RPRRR PMs

(1) Input topology matrices of the PM

$$
\begin{aligned}
& \text { Topology matrix of the first (and the fourth) leg is, } \mathrm{L}_{\mathrm{i}}=\left[\begin{array}{lllll}
8 & 2 & 1 & 0 & 0 \\
2 & 9 & 2 & 0 & 0 \\
1 & 2 & 8 & 0 & 0 \\
0 & 0 & 0 & 8 & 4 \\
0 & 0 & 0 & 4 & 8
\end{array}\right]_{(1)},(\mathrm{i}=1,4) . \\
& \text { Topology matrix of the second (and the third) leg is, } \mathrm{L}_{\mathrm{i}}=\left[\begin{array}{llllll}
8 & 2 & 1 & 0 & 0 \\
2 & 9 & 2 & 0 & 0 \\
1 & 2 & 8 & 0 & 0 \\
0 & 0 & 0 & 8 & 4 \\
0 & 0 & 0 & 4 & 8
\end{array}\right]_{(2)},(\mathrm{i}=2,3) \text {. }
\end{aligned}
$$

where, the subscript 1 and 2 represent the No. of intersection point of the last two revolute joints in the legs.

Topology matrices of two virtual legs on the two platforms $L_{M P}$ and $L_{B P}$ are $L_{M P}=\left[\begin{array}{llll}8 & 0 & 0 & 4 \\ 0 & 8 & 4 & 0 \\ 0 & 4 & 8 & 0 \\ 4 & 0 & 0 & 8\end{array}\right]$ and $\mathrm{L}_{\mathrm{BP}}=\left[\begin{array}{llll}8 & 1 & 2 & 2 \\ 1 & 8 & 2 & 2 \\ 2 & 2 & 8 & 1 \\ 2 & 2 & 1 & 8\end{array}\right]$, respectively.

Number of legs $v+1=4$.

(2) Select the point $\mathrm{O}_{1}{ }^{\prime}$ on the moving platform as the base point $\left(\mathrm{O}^{\prime}\right)$.

(3) Calculate POC matrix of legs $\mathrm{M}_{\mathrm{L} 1}, \mathrm{M}_{\mathrm{L} 2}, \mathrm{M}_{\mathrm{L} 3}$ and $\mathrm{M}_{\mathrm{L} 4}$.

(1) Recognize and sequentially extract planar and spherical sub-chains: one $G_{3}$ sub-chain $R \perp P / / R$ and one $S_{2}$ sub-chain $R^{*} R$ respectively.

(2) Generate the POC supplemented matrices of $G_{3}$ and $S_{2}$ as the follows,

For the first (or the fourth) leg: $\mathrm{M}_{\mathrm{G} 3}=\left[\begin{array}{llllll}2 & 0 & 0 & 0 & 0 & 0 \\ 1 & 0 & 0 & 0 & 0 & 0\end{array}\right]$ and $\mathrm{M}_{\mathrm{S} 2}=\left[\begin{array}{llllll}0 & 0 & 0 & 0 & 0 & 0 \\ 0 & 0 & 0 & 1 & 1 & 0\end{array}\right]+\left[\begin{array}{c}0 \\ \# 1\end{array}\right]$.

For the second (or the third) leg: $\mathrm{M}_{\mathrm{G} 3}=\left[\begin{array}{llllll}2 & 0 & 0 & 0 & 0 & 0 \\ 1 & 0 & 0 & 0 & 0 & 0\end{array}\right]$ and $\mathrm{M}_{\mathrm{S} 2}=\left[\begin{array}{llllll}0 & 0 & 0 & 0 & 0 & 0 \\ 0 & 0 & 0 & 1 & 1 & 0\end{array}\right]+\left[\begin{array}{c}2 \\ \# 2\end{array}\right]$.

Among which, subscript 1 (2) indicates the intersecting points of the last two revolute joints, the number 2 means two derivative translational outputs in the plane perpendicular to the line connecting point $\mathrm{O}^{\prime}$ and $\mathrm{O}_{2}{ }^{\prime}$.

(3) Carry out "or" operation on POC matrices

To the first (and the fourth) leg: $\mathrm{M}_{\mathrm{Li}}=\left[\begin{array}{llllll}2 & 0 & 0 & 0 & 0 & 0 \\ 3 & 0 & 0 & 0 & 0 & 0\end{array}\right]_{(1)}$, so $\xi_{\mathrm{r}}^{0}=3, \xi_{\mathrm{t}}^{0}=2$ and $\rho_{\mathrm{i}}=\mathrm{OO}_{1}{ }^{\prime}(\mathrm{i}=1,4)$.

To the second (and the third) leg: $\mathrm{M}_{\mathrm{Li}}=\left[\begin{array}{llllll}2 & 0 & 0 & 0 & 0 & 0 \\ 3 & 0 & 0 & 0 & 0 & 0\end{array}\right]+\left[\begin{array}{c}2 \\ \# 2\end{array}\right]$, so $\xi_{\mathrm{r}}^{0}=3, \xi_{\mathrm{t}}^{0}=3$ and $\rho_{\mathrm{i}}=\mathrm{O}^{\prime} \mathrm{O}_{2}{ }^{\prime}(\mathrm{i}=2,3)$.

(4) Then $\mathrm{M}_{\mathrm{Li}}=\left[\begin{array}{llllll}2 & 0 & 0 & 0 & 0 & 0 \\ 3 & 0 & 0 & 0 & 0 & 0\end{array}\right]+\left[\begin{array}{c}0 \\ \# 1\end{array}\right](\mathrm{i}=1,4)$, and $\mathrm{M}_{\mathrm{Li}}=\left[\begin{array}{llllll}3 & 0 & 0 & 0 & 0 & 0 \\ 3 & 0 & 0 & 0 & 0 & 0\end{array}\right](\mathrm{i}=2,3)$, i.e., $\xi_{\mathrm{ti}}=2, \mathrm{e}_{\mathrm{i}}=\perp\left(\mathrm{R}_{\mathrm{i} 1}\right)$ $(\mathrm{i}=1,4)$, and $\xi_{\mathrm{ii}}=3(\mathrm{i}=2,3)$, and $\xi_{\mathrm{ri}}=3(\mathrm{i}=1 \sim 4)$. 
(4) Calculate the sum of all joints in this PM: $f_{(1-3)}=5+5+5+5=20$.

(5) Calculate the POC matrix $\mathrm{M}_{\mathrm{pa}\left(1^{\sim} \mathrm{j}\right)}$ of sub-PM $\mathrm{M}_{\left(1^{\sim} \mathrm{j}\right)}$ constituted by the front $\mathrm{j}$ legs in parallel.

- Set $\mathrm{M}_{\mathrm{pa}(1 \sim 1)}=\mathrm{M}_{\mathrm{L} 1}$

- Calculate the $P O C$ matrix $\mathrm{M}_{\mathrm{pa}(1 \sim 2)}$ of sub-PM $\mathrm{M}_{(1 \sim 2)}$ with $\mathrm{M}_{\mathrm{pa}(1 \sim 2)}=\mathrm{M}_{\mathrm{pa}(1 \sim 1)} \cap \mathrm{M}_{\mathrm{L} 2}$.

(1) $\xi_{\mathrm{t} 1}=2$, and $\xi_{\mathrm{t} 2}=3$, as shown in Table $5, \xi_{\mathrm{t}(1 \sim 2)}=2$, and $\mathrm{e}_{\left(1^{\sim} \sim 2\right)}=\mathrm{e}_{1}$.

(2) $\xi_{\mathrm{r} 1}=3, \xi_{\mathrm{r} 2}=3$, as shown in Table $6, \xi_{\mathrm{r}(1 \sim 2)}=\xi_{\mathrm{r} 1}=3$.

(3) So, $\mathrm{M}_{\mathrm{pa}(1 \sim 2)}=\left[\begin{array}{c}G_{1} \\ H_{1}\end{array}\right]$.

- Calculate the POC matrix $M_{p a(1 \sim 3)}$ of sub-PM $M_{(1 \sim 3)}$ with $M_{p a(1 \sim 3)}=M_{p a(1 \sim 2)} \cap M_{L 3}$.

(1) $\xi_{t(1 \sim 2)}=2, e_{(1 \sim 2)}=e_{1}$, and $\xi_{t 3}=3$, as shown in Table 5, $\xi_{t(1 \sim 3)}=2$, and $e_{(1 \sim 3)}=e_{1}$.

(2) $\xi_{\mathrm{r}(1 \sim 2)}=3, \xi_{\mathrm{r} 3}=3$, as shown in Table 6, $\xi_{\mathrm{r}(1 \sim 3)}=3$.

(3) So, $\mathrm{M}_{\mathrm{pa}(1 \sim 3)}=\left[\begin{array}{l}G_{1} \\ H_{1}\end{array}\right]$.

(6) Calculate the number of the independent displacement equations $\xi_{\mathrm{L} j}$ of $\operatorname{SLC}(\mathrm{j}=1 \sim 2)$.

- Calculate $\xi_{L 1}$ of the first loop SLC 1 concatenated by the $1^{\text {st }}$ leg and the $2^{\text {nd }}$ leg.

(1) $\xi_{\mathrm{t} 1}=2$, and $\xi_{\mathrm{t} 2}=3$, as shown in Table 7, $\xi_{\mathrm{Lt} t}=3(\mathrm{i}=1,2)$.

(2) $\xi_{\mathrm{r} 1}=3, \xi_{\mathrm{r} 2}=3$, as shown in Table 8, then $\xi_{\mathrm{Lrr}}=3(\mathrm{i}=1,2)$.

(3) So, $\xi_{L 1}=\xi_{L 1 t}+\xi_{L 1 r}=3+3=6$.

- Calculate the number of the independent displacement equations $\xi_{L_{2}}$ of $\operatorname{SLC}_{2}\left(\xi_{L_{2}}=\operatorname{dim}\left(M_{\mathrm{pa}(1 \sim 2)} \cup \mathrm{M}_{\mathrm{L} 3}\right)\right)$

(1) $\xi_{\mathrm{t} 3}=3$, and $\mathrm{e}_{(1 \sim 2)}=\mathrm{e}_{1}$, as shown in Table $7, \xi_{\mathrm{L2t}}=3$.

(2) $\xi_{\mathrm{r} 3}=3$, and $\xi_{\mathrm{r}(1 \sim 2)}=3$, as shown in Table $8, \xi_{\mathrm{L} 2 \mathrm{r}}=3$.

(3) Thus, $\xi_{\mathrm{L} 2}=\xi_{\mathrm{L2t}}+\xi_{\mathrm{L2r}}=3+3=6$.

- Calculate the number of the independent displacement equations $\xi_{\mathrm{L} 2}$ of $\operatorname{SLC}_{3}\left(\xi_{\mathrm{L}_{3}}=\operatorname{dim}\left(\mathrm{M}_{\mathrm{pa}\left(1^{1} 3\right)} \cup \mathrm{M}_{\mathrm{L} 4}\right)\right)$

(1) $\xi_{t 4}=2, e_{(1 \sim 3)}=e_{1}$, and $e_{(1 \sim 3)} H \mathrm{e}_{4}\left(\perp R_{41)}\right.$, as shown in Table 7, $\varepsilon_{L 3 t}=3$.

(2) $\xi_{\mathrm{r} 4}=3$, and $\xi_{\mathrm{r}(1 \sim 3)}=3$, as shown in Table $8, \xi_{\mathrm{Lzr}}=3$.

(3) Thus, $\xi_{L 2}=\xi_{L 2 t}+\xi_{L 2 r}=3+3=6$.

(7) Get the number of DOF: $\mathrm{F}=\mathrm{f}_{(1 \sim 4)}-\sum_{i=1}^{3} \xi_{\mathrm{i}}=20-(6+6+6)=2$

(8) Get the mobility property: $\mathrm{M}_{\mathrm{pa}}=\mathrm{M}_{\mathrm{pa}(1-3)} \cap \mathrm{M}_{\mathrm{L4}}=\left[\begin{array}{c}G_{1} \\ H_{1}\end{array}\right] \mathrm{I}\left[\begin{array}{c}G_{4} \\ H_{4}\end{array}\right]=\left[\begin{array}{c}\left(\perp R_{11}\right) \mathrm{I}\left(\perp R_{41}\right) \\ H_{1}\end{array}\right]$.

The automatically generated result is shown in Fig.11 and the result is consistent with the result in [18]. Since DOF of this PM is 2, the POC has two independent elements, and the other two elements are non-independent. Without loss of generality, one translation along the common perpendicular of axes of $R_{11}$ and $R_{41}$, and one rotation around $\mathrm{O}_{1}{ }^{\prime}-\mathrm{O}_{2}^{\prime}$ are selected as two independent elements, and the $\mathrm{POC}$ is written as $\mathrm{Mpa}=\left[\begin{array}{c}t_{\left(\perp R_{11}, R_{41}\right)}^{1} \\ r_{\left(o_{1}^{\prime}-o_{2}^{\prime}\right)}^{1} \mathrm{U}\left\{r^{2}\right\}\end{array}\right]$ in [18].

(9) Discussion

If the intersecting points of $\mathrm{O}_{1}{ }^{\prime}$ and $\mathrm{O}_{2}{ }^{\prime}$ coincide together, a new PM is obtained in Fig.10(b). Select the interseting point as the base point, and follow above steps (1) to (8). It is easy to determine that the POC is $\mathrm{M}_{\mathrm{pa}}=\left[\begin{array}{cc}G_{1} \mathrm{I} & G_{4} \\ H_{1}\end{array}\right]$ and DOF is four.

Although this POC has the same form as that of the original PM, it should be noted that since DOF is four, the $P O C$ has four independent elements. So, the PM has three independent rotations and one independent translation along the common perpendicular of axis of $R_{11}$ and axis of $R_{41}$. 


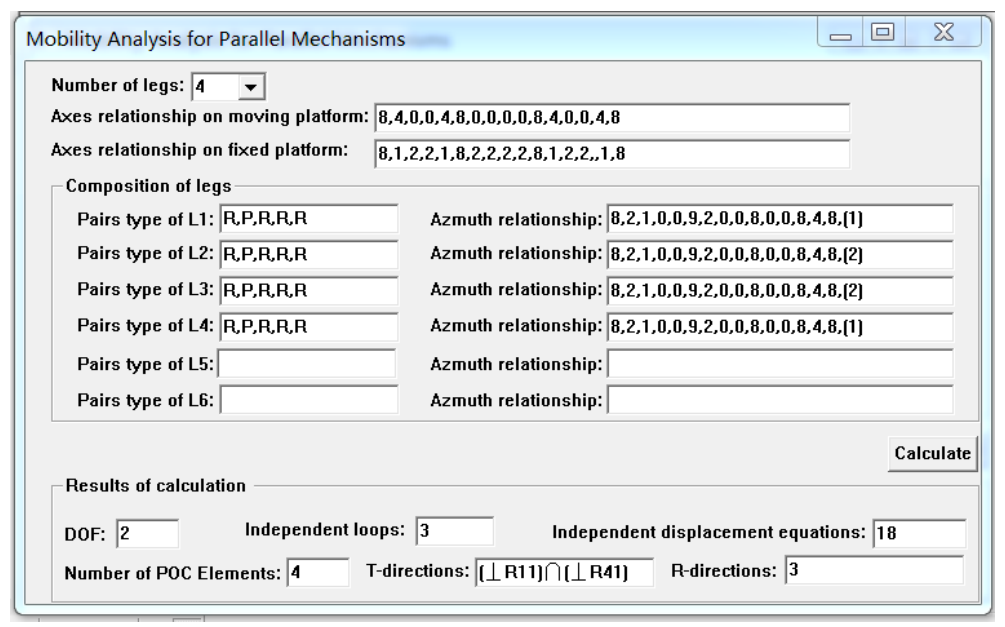

Fig. 11. Mobility analysis of the 4-RPRRR PM shown in Fig.10(a)

\section{Conclusions}

In this paper, a computer-aided method for mobility analysis of PMs is proposed. A matrix representation mapping the topological structure of a leg or a PM into their POC is established. This matrix includes not only the dimension of translation/rotation output, but also indicates the orientation of the output motion. By extracting planar and spherical sub-chains, calculating the POC for legs transforms into logical bitwise "or" operation of matrices. Algorithmic rules for computing the POCs of PMs are established without manual intervention. Based on the proposed method, the process of mobility analysis is performed by a computer program, and a humancomputer interactive interface has been developed for potential designers. four typical examples are provided in detail to show the effectiveness of the developed algorithms. The relevant study of this paper is expected to benefit the automation and computerization of the mechanical creative design and analysis.

For the "paradoxical" mechanisms that the software mentioned here currently can not solve, the future work will concentrate on proposing the digital model of the dimensional constraint parameters (link length, axial distance between two joint axes, etc.) of the PMs, and then focus on establishing the mapping relationship between the model and the POC. Further, the automatic analysis for another important topological properties of PMs such as coupling degree will be presented.

\section{ACKNOWLEDGMENT}

This work was supported by the National Natural Science Foundation of China (Grants No. 51975062, No. 51475050 and No. 51375062). The authors express their gratitude to Prof. Manfred Husty for his kind and fine revision of this manuscript.

\section{References}

[1] Rey L, Clavel R . The Delta Parallel Robot, Parallel Kinematic Machines, Springer London(1999), doi:10.1007/978-1-44710885-6_29.

[2] G. Gogu, Chebyshev-Grubler-Kutzbach's criterion for mobility calculation of multi-loop mechanisms revisited via theory of linear transformations, Eur. J. Mech. A-Solid 24(3) (2005) 427-441, doi:10.1016/j.euromechsol.2004.12.003.

[3] G. Gogu, Mobility of mechanisms: a critical review, Mech. Mach. Theory 40(9) (2005) 1068-1097, doi: 10.1016/ j.mechmachtheory.2004.12.014.

[4] R. Alizade, C Bayram, E. Gezgin, Structural synthesis of serial platform manipulators, Mech. Mach. Theory 42 (2007) 580599, doi:10.1016/j.mechmachtheory.2006.05.005.

[5] J.S. Dai, D. Li, Q. Zhang, et al., Mobility analysis of a complex structured ball based on mechanism decomposition and equivalent screw system analysis, Mech. Mach. Theory 39(4) (2004) 445-458, doi:10.1016/j.mechmachtheory.2003.12.004.

[6] J.S. Dai, Z. Huang, H. Lipkin, Mobility of overconstrained parallel mechanisms, ASME J. Mech. Design 128(1) (2006) 220229, doi:10.1115/1.1901708. 
[7] X.W. Kong, C. Gosselin, Type synthesis of 3T1R 4-DOF parallel manipulators based on screw theory, IEEE Trans. Robot. Auto. 20(2) (2004) 181-190, doi:10.1109/tra.2003.820853.

[8] Z. Huang, J.F. Liu, D.X. Zeng, A general methodology for mobility analysis of mechanisms based on constraint screw theory, Science in China Series E-Technological Sciences (in Chinese) 39(1) (2009) 84-93, https://doi.org/10.1360/ze2009-39-1-84.

[9] Z. Huang, J.F. Liu, Q.C. Li, A unified methodology for mobility analysis based on screw theory, Smart Devices and Machines for Advanced Manufacturing, Springer Publishing (2008) 49-78, doi:10.1007/978-1-84800-147-3_3.

[10] A.A. Nazari, H. Ayyub, B. Majid, Screw theory-based mobility analysis and projection-based kinematic modeling of a 3-CRRR parallel manipulator, Journal of the Brazilian Society of Mechanical Sciences and Engineering 40(7) (2018) 357-373, doi:10.1007/s40430-018-1277-3.

[11] J.S. Zhao, F.L. Chu, Z.J. Feng, Mobility of spatial parallel manipulators, i-Tech Education Publishing, Vienna Austria 2008, doi:10.5772/5443.

[12] W.A. Cao, Digital Type synthesis theory of spatial multi-loop coupled mechanisms(Doctoral dissertation), Yanshan University (2014), http://cdmd.cnki.com.cn/Article/CDMD-10216-1016704802.htm.

[13] P. Fanghella, C. Galletti, Mobility analysis of single-loop kinematic chains: an algorithmic approach based on displacement groups, Mech. Mach. Theory 29(8) (1994) 1187-1204, doi:10.1016/0094-114x(94)90009-4.

[14] J.M. Herve, The Lie group of rigid body displacements, a fundamental tool for mechanism design, Mech. Mach. Theory 34(5) (1999) 719-730, doi:10.1016/S0094-114X(98)00051-2.

[15] J.M. Rico, J. Gallardo, B. Ravani, Lie algebra and the mobility of kinematic chains, J. Robotic Syst. 20(8) (2003) 477-499, doi:10.1002/rob.10099.

[16] J.M. Rico, B. Ravani, On mobility analysis of linkages using group theory, ASME J. Mech. Design 125(1) (2003) 70-80, doi.org/10.1115/1.1541628.

[17] A. Daniel, J. Rico, and J. Gallardo, Computer implementation of an improved Kutzbach-Grubler mobility criterion, In ASME IDETC-CIE, Montreal, Quebec, September 29-October 2, (2002) 549-557, doi:10.1115/DETC2002/DAC-34093.

[18] T.L. Yang, A. Liu, H. Shen, et al., Topology design of robot mechanisms, Springer Publishing (2018), doi:10.1007/978-981-105532-4.

[19] T.L. Yang, D.J. Sun, A general degree of freedom formula for parallel mechanisms and multiloop spatial mechanisms, J. Mechanisms Robotics. 4(1) (2012) 1-17, https://doi.org/10.1115/1.4005526.

[20] T. Yang, A. Liu, Y. Luo, et al., On the correctness and strictness of the position and orientation characteristic equation for topological design of robot mechanisms, J. Mechanisms Robotics 5(2) (2013) 1-18, https://doi.org/10.1115/1.4023871.

[21] T. Yang, A. Liu, Y. Luo, et al., Position and orientation characteristic equation for topological design of robot mechanisms, ASME J. Mech. Design 131(2) (2009) 1-17, doi:10.1115/DETC2008-49074.

[22] T.L. Yang, H. Shen, A. Liu, et al., Review of the formulas for degree of freedom in the past ten years, Journal of Mechanical Engineering 51(13) (2015) 69-80, doi:10.3901/JME.2015.13.069.

[23] Q. Jin, T.L. Yang, Theory for topology synthesis of parallel manipulators and its application to three-dimension-translation parallel manipulators, J. Mech. Design 126(4) (2004) 625-639, doi:10.1115/1.1758253.

[24] J. Angles, C. Gosselin, On the determination of the degree of freedom of simple and complex kinematic chains, Transactions of the Canadian Society for Mechanical Engineering 12(4) (1988) 219-226, https://doi.org/10.1139/tcsme1988-0031.

[25] M.L. Husty, H.P. Schröcker, A proposal for a new definition of the degree of freedom of a mechanism, Interdisciplinary Applications of Kinematics (2012) 109-118, doi:10.1007/978-94-007-2978-0-8.

[26] Xinming Huo, Tao Sun, Yimin Song, A geometric algebra approach to determine motion/constraint, mobility and singularity of parallel mechanism, Mech. Mach. Theory, (116) (2017) 273-293, https://doi.org/10.1016/j.2017.06.005.

[27] Xinming Huo, Tao Sun, Yimin Song, et al., An analytical approach to determine motions/constraints of serial kinematic chains based on Clifford algebra, Proceedings of the Institution of Mechanical Engineers, Part C: Journal of Mechanical Engineering Science, 231(7) (2017) 1324-1338, doi:10.1177/0954406216643561.

[28] Shuofei Yang, Tao Sun, Tian Huang, Type synthesis of parallel mechanisms having 3T1R motion with variable rotational axis, Mech. Mach. Theory, (109) (2017) 220-230, https://doi.org/10.1016/j.mechmachtheory.2016.11.005.

[29] X. Zhu, H. Shen, C. Wu, et.al., Automatic mobility analysis of parallel mechanisms based on position and orientation characteristic equation. Part II: mobility analysis and examples, The IFToMM Symposium, Cham, USA, (2019) 2789-2798, https://link.springer.com/chapter/10.1007/978-3-030-20131-9_276.

[30] X. Zhu, H. Shen, C. Wu, et al., Automatic mobility analysis of parallel mechanisms: an algorithm approach based on position and orientation characteristic equations, In ASME 2019 International Design Engineering Technical Conferences and Computers and Information in Engineering Conference. American Society of Mechanical Engineers Digital Collection, 2019, https://arxiv.org/abs/1907.08428.

[31] H. Shen, T.L. Yang, J. Li, D. Zhang, J. Deng and A. Liu, Evaluation of Topological Properties of Parallel Manipulators Based on the Topological Characteristic Indexes, Robotica,DOI:S0263574719001528,19.11,2019 